\title{
A Schrödinger Equation for Solving the Riemann Hypothesis
}

\author{
Frederick Ira Moxley III $^{1}$ \\ ${ }^{1}$ Hearne Institute for Theoretical Physics, Department of Physics $\& 3$ Astronomy, \\ Louisiana State University, Baton Rouge, Louisiana 70803-4001, USA
}

\begin{abstract}
The Hamiltonian of a quantum mechanical system has an affiliated spectrum. If this spectrum is the sequence of prime numbers, a connection between quantum mechanics and the nontrivial zeros of the Riemann zeta function can be made. In this case, the Riemann zeta function is analogous to chaotic quantum systems, as the harmonic oscillator is for integrable quantum systems. Such quantum Riemann zeta function analogies have led to the Bender-Brody-Müller (BBM) conjecture, which involves a non-Hermitian Hamiltonian that maps to the zeros of the Riemann zeta function. If the BBM Hamiltonian can be shown to be Hermitian, then the Riemann Hypothesis follows. As such, herein we perform a symmetrization procedure of the BBM Hamiltonian to obtain a unique Hermitian Hamiltonian that maps to the zeros of the analytic continuation of the Riemann zeta function, and provide an analytical expression for the eigenvalues of the results. The holomorphicity of the resulting eigenvalues is examined. Moreover, a second quantization of the resulting Schrödinger equation is performed, and a convergent solution for the nontrivial zeros of the analytic continuation of the Riemann zeta function is obtained. Finally, from the holomorphicity of the eigenvalues it is shown that the real part of every nontrivial zero of the Riemann zeta function converges at $\sigma=1 / 2$.

Keywords: quantum chaos; Riemann hypothesis; eigenspectra
\end{abstract}

\section{INTRODUCTION}

The unification of number theory with quantum mechanics has been the subject of many research investigations [1]5]. It has been proven that an infinitude of prime numbers exist [6]. In Ref. [7, it was shown that the eigenvalues of a Bender-Brody-Müller (BBM) Hamiltonian operator correspond to the nontrivial zeros of the Riemann zeta function. If the Riemann hypothesis is correct [8, the zeros of the Riemann zeta function can be considered as the spectrum of an operator $\hat{R}=\hat{I} / 2+i \hat{H}$, where $\hat{H}$ is a self-adjoint Hamiltonian operator [5, 9], and $\hat{I}$ is identity. Hilbert proposed the Riemann Hypothesis as the eighth problem on a list of significant mathematics problems [10. Although the BBM Hamiltonian is pseudo-Hermitian [11, it is consistent with the Berry-Keating conjecture [12 14, which states that when $\hat{x}$ and $\hat{p}$ commute, the Hamiltonian reduces to the classical $H=2 x p$. Berry, Keating and Connes proposed the classical Hamiltonian in an effort to map the Riemann zeros to the Hamiltonian spectrum. More recently, the classical Berry-Keating Hamiltonians were quantized, and were found to contain a smooth approximation of the Riemann zeros [15, 16. This reformulation was found to be physically equivalent to the Dirac equation in Rindler spacetime [17. Herein, the eigenvalues of the BBM Hamiltonian are taken to be the imaginary parts of the nontrivial zeroes of the analytical continuation of the Riemann zeta function

$$
\zeta(s)=\frac{1}{1-2^{1-s}} \cdot \sum_{n=1}^{\infty} \frac{(-1)^{n-1}}{n^{s}},
$$

where the complex number $s=\sigma+i t$, and $\Re(s)>0$. The idea that the imaginary parts of the zeros of Eq. (1) are given by a self-adjoint operator was conjectured by Hilbert and Pólya [18. Hilbert and Pólya asserted that the nontrivial zeros of Eq. (1) can be considered as the spectrum of a self-adjoint operator in a suitable Hilbert space. The Hilbert-Pólya conjecture has also found applications in quantum field theories [19]. The Riemann Hypothesis states that the zeros of Eq. (1) on $0 \leq \sigma<1$ have real part equal to $1 / 2$ [8, 20. In Ref. [21, Hardy proved that infinitely many zeros are located at $\sigma=1 / 2$. According to the Prime Number Theorem [22, 23, no zeros of Eq. (1) can exist at $\sigma=1$. In this paper we present a Schrödinger equation that maps to the nontrivial zeros of the Riemann zeta function, and evaluate the convergence of the expression by imposing a normalization constraint on the density. A self-adjoint Hamiltonian is derived from the BBM Hamiltonian, and a second quantization of the resulting Schrödinger equation is performed to obtain the equations of motion. We study the nontrivial zeros of the Riemann zeta function by taking the expectation values of the resulting Schrödinger equation to show that the real part of every nontrivial zero of the analytic continuation of the Riemann zeta function converges at $\sigma=1 / 2$. Finally we obtain a general solution to the Riemann zeta Schrödinger equation by performing a similarity transformation. 


\section{RIEMANN ZETA SCHRÖDINGER EQUATION}

We consider the eigenvalues of the Hamiltonian

$$
\hat{H}=\frac{1}{1-e^{-i \hat{p}}}(\hat{x} \hat{p}+\hat{p} \hat{x})\left(1-e^{-i \hat{p}}\right),
$$

where $\hat{p}=-i \hbar \partial_{x}, \hbar=1$, and $\hat{x}=x$. In Ref. [7, it is conjectured that if the Riemann hypothesis is correct, the eigenvalues of Eq. (2) are non-degenerate. Next, we let $\Psi_{s}(x)$ be an eigenfunction of Eq. (2) with an eigenvalue $t=i(2 s-1)$, such that

$$
\hat{H}\left|\Psi_{s}(x)\right\rangle=t\left|\Psi_{s}(x)\right\rangle
$$

and $x \in \mathbb{R}^{+}, s \in \mathbb{C}$. Solutions to Eq. (3) are given by the analytic continuation of the Hurwitz zeta function

$$
\begin{aligned}
\left|\Psi_{s}(x)\right\rangle & =-\zeta(s, x+1) \\
& =-\Gamma(1-s) \frac{1}{2 \pi i} \oint_{C} \frac{z^{s-1} e^{(x+1) z}}{1-e^{z}} d z
\end{aligned}
$$

on the positive half line $x \in \mathbb{R}^{+}$with eigenvalues $i(2 s-1), s \in \mathbb{C}, \Re(s) \leq 1$, the contour $C$ is a loop around the negative real axis, and $\Gamma$ is the Euler gamma function for $\Re(s)>0$

$$
\Gamma(s)=\int_{0}^{\infty} x^{s-1} e^{-x} d x .
$$

As $-\left|\Psi_{s}(x=1)\right\rangle$ is $1-\zeta\left(s^{*}\right)$, this implies that $s$ belongs to the discrete set of zeros of the Riemann zeta function when $s^{*}=\sigma-i t$, and as $-\left|\Psi_{s}(x=-1)\right\rangle$ is $\zeta(s)$, this implies that $s$ belongs to the discrete set of zeros of the Riemann zeta function when $s=\sigma+i t$. From inserting Eq. (3) into Eq. (2), we have the relation

$$
\frac{1}{1-e^{-i \hat{p}}}(\hat{x} \hat{p}+\hat{p} \hat{x})\left(1-e^{-i \hat{p}}\right)\left|\Psi_{s}(x)\right\rangle=t\left|\Psi_{s}(x)\right\rangle .
$$

Given that Eq. (2) is not Hermitian, it is useful to symmetrize the system. This can be accomplished by letting

$$
\begin{aligned}
\left|\phi_{s}(x)\right\rangle & =\left[1-\exp \left(-\partial_{x}\right)\right]\left|\Psi_{s}(x)\right\rangle \\
& =\hat{\Delta}\left|\Psi_{s}(x)\right\rangle \\
& =\left|\Psi_{s}(x)\right\rangle-\left|\Psi_{s}(x-1)\right\rangle
\end{aligned}
$$

and defining a shift operator

$$
\hat{\Delta} \equiv 1-\exp \left(-\partial_{x}\right)
$$

For $s>0$ the only singularity of $\zeta(s, x)$ in the range of $0 \leq x \leq 1$ is located at $x=0$, behaving as $x^{-s}$. More specifically,

$$
\zeta(s, x+1)=\zeta(s, x)-\frac{1}{x^{s}}
$$

with $\zeta(s, x)$ finite for $x \geq 1$ [25]. As such, it can be seen from Eq. (7) that the eigenfunction

$$
\left|\phi_{s}(x)\right\rangle=\frac{1}{x^{s}}
$$

Upon inserting Eq. (7) into Eq. (6) we obtain

$$
-i\left[x \partial_{x}+\partial_{x} x\right]\left|\phi_{s}(x)\right\rangle=t\left|\phi_{s}(x)\right\rangle
$$


Let $\mathscr{H}$ be a Hilbert space, and from Eq. 11] we have the Hamiltonian operator

$$
\begin{aligned}
\hat{H} & =-i \hbar\left[x \partial_{x}+\partial_{x} x\right] \\
& =-i \hbar\left[2 x \partial_{x}+1\right],
\end{aligned}
$$

for $x \in \mathbb{R}$ acting in $\mathscr{H}$, such that

$$
\langle\hat{H} f, g\rangle=\langle f, \hat{H} g\rangle \forall f, g \in \mathscr{D}(\hat{H}) .
$$

Restricting $x \in \mathbb{R}^{+}$, Eq. 12 is then written

$$
\hat{H}=-2 i \hbar \sqrt{x} \partial_{x} \sqrt{x}
$$

where $s \in \mathbb{C}$, and $x \in \mathbb{R}^{+}$. For the Hamiltonian operator as given by Eq. 14, the Hilbert space is $\mathscr{H}=L^{2}(1, \infty)$ [26 28. We then impose on Eq. (14) the following minimal requirements, such that its domain is not too artificially restricted.

i $\hat{H}$ is a symmetric (Hermitian) linear operator;

ii $\hat{H}$ can be applied on all functions of the form

$$
g(x, s)=P(x, s) \exp \left(-\frac{x^{2}}{2}\right),
$$

where $P$ is a polynomial of $x$ and $s$. Here, it should be pointed out that $\hat{H}=\hat{T}+\hat{V}$, and from Eq. (12), it can be seen that $\hat{T}=-2 i \hbar x \partial_{x}, \hat{V}=-i \hbar$. From (ii), $\hat{V} g(x, s)$ must belong to the Hilbert space $\mathscr{H}=L^{2}$ defined over the space $x$. This is guaranteed as

$$
|-i \hbar| \leq \hbar
$$

where $\hbar$ is the reduced Planck constant or Dirac constant. The domain $\mathscr{D}_{\hat{V}}$ of the potential energy $\hat{V}$ consists of all $\phi \in \mathscr{H}$ for which $\hat{V} \phi \in \mathscr{H}$. As such, $\hat{V}$ is self-adjoint. It is not necessary to specify the domain of Eq. 14, as it is only necessary to admit that Eq. (14) is defined on a certain $\mathscr{D}_{\hat{H}}$ such that (i) and (ii) are satisfied. If we denote by $\mathscr{D}_{1}$ the set of all functions in Eq. 15 , then (ii) implies that $\mathscr{D}_{\hat{H}} \supseteq \mathscr{D}_{1}$. By letting $\hat{H}_{1}$ be the contraction of $\hat{H}$ with domain $\mathscr{D}_{1}$, i.e., $\hat{H}$ is an extension of $\hat{H}_{1}$, and letting $\tilde{H}_{1}$ be the closure of $\hat{H}$, it can be seen that $\tilde{H}_{1}$ is self-adjoint. Since $\hat{H}$ is symmetric and $\hat{H} \supseteq \hat{H}_{1}$, i.e., $\hat{H}$ is an extension of $\hat{H}_{1}$, it follows that $\tilde{H}=\tilde{H}_{1}$ and $\hat{H}$ is essentially self-adjoint, where $\tilde{H}$ is the unique self-adjoint extension [29]. Other than eigenfunctions $\phi_{s}(x)$ in configuration space as seen in Eq. (10), it is useful to represent eigenfunctions in momentum space $\Phi_{s}(p)$. The transformation between configuration space eigenfunctions and momentum space eigenfunctions can be obtained via Plancherel transforms [30, where the one-to-one correspondence $\phi_{s}(x) \rightleftharpoons \Phi_{s}(p)$ is linear and isometric.

Theorem 1. The eigenstate $\phi_{s}(x): \mathbb{X} \rightarrow \mathbb{C}$ is measurable. That is, $\phi_{s}(x)=\phi_{\sigma}(x)+i \phi_{t}(x)$ where $\phi_{\sigma}(x), \phi_{t}: \mathbb{X} \rightarrow$ $(-\infty,-1] \cup[1, \infty)$ are measurable.

Proof.

$$
\|\phi\|^{2} \equiv \int_{-\infty}^{-1}\left|\phi_{s}(x)\right|^{2} d x+\int_{1}^{\infty}\left|\phi_{s}(x)\right|^{2} d x=\int_{-\infty}^{-1}\left|\Phi_{s}(p)\right|^{2} d p+\int_{1}^{\infty}\left|\Phi_{s}(p)\right|^{2} d p \equiv\|\Phi\|^{2},
$$

from which

$$
\begin{aligned}
\Phi_{s}(p) & =\frac{1}{2 \pi^{3 / 2}} \int_{-\infty}^{\infty} \phi_{s}(x) \exp (-i p x) d x \\
& =\frac{e^{-\frac{1}{2} i \pi s}(\operatorname{sgn}(p)+1) \sin (\pi s) \Gamma(1-s)|p|^{s-1}}{2 \pi^{3 / 2}}, 0<\Re(s)<1 \\
\phi_{s}(x) & =\frac{1}{2 \pi^{3 / 2}} \int_{-\infty}^{\infty} \Phi_{s}(p) \exp (i p x) d p=\frac{1}{x^{s}}
\end{aligned}
$$


and

$$
\|\phi\|^{2}=\|\Phi\|^{2}=\frac{(-1)^{-2 \sigma}}{2 \sigma-1}+\frac{1}{2 \sigma-1},
$$

where $\sigma>1 / 2$. Interestingly, it can be seen that

$$
\lim _{\sigma \rightarrow 1 / 2}\left[\frac{(-1)^{-2 \sigma}}{2 \sigma-1}+\frac{1}{2 \sigma-1}\right]=i \pi=\log (x=-1) .
$$

Then,

$$
\phi_{s}(x) \in L^{2}(\mu) \Longleftrightarrow \int_{\mathbb{X}}\left|\phi_{\sigma}(x)\right|^{2} d \mu<\infty
$$

and

$$
\int_{\mathbb{X}}\left|\phi_{t}(x)\right|^{2} d \mu<\infty
$$

Eqs. (18) and (19) are two vector representations of the same Hilbert space $\mathscr{H}$. From Eq. (12), it can be seen that

$$
\hat{T}=-2 i \hbar x \partial_{x}
$$

such that we define a multiplicative operator $\hat{T}_{0}$ in momentum space $\left(\hat{T}_{0} \Phi_{s}\right)(p)=\hat{T}_{0}(p) \Phi_{s}(p)$, where

$$
\hat{T}_{0}(p)=2 \hat{x} \hat{p} .
$$

Here, it should be pointed out that as $\hat{x}=i \hbar d / d p$, Eq. 25) reduces to

$$
\hat{T}_{0}(p)=2 i \hbar,
$$

and Eq. 12 is then rewritten in momentum space as $\hat{H}(p)=i \hbar$. The domain $\mathscr{D}_{0}$ of $\hat{T}_{0}$ is defined as the set of all functions $\Phi_{s}(p) \in \mathscr{H}$ such that $\hat{T}_{0}(p) \Phi_{s}(p) \in \mathscr{H}$. As such, $\hat{T}_{0}$ is definitively self-adjoint. From Eq. 15 we have defined the set $\mathscr{D}_{1}$ of functions in configuration space. From the Plancherel transform [30] of Eq. 115], we obtain the set $\mathscr{D}_{1}$ of functions in momentum space having the form

$$
G(p, s)=P(p, s) \exp \left(-\frac{p^{2}}{2}\right),
$$

where $P$ is a polynomial of $p$ and $s$. Eqs. (18) and (19) are true if $\phi_{s}(x) \in \mathscr{D}_{1}$ or $\Phi_{s}(p) \in \mathscr{D}_{1}$ and since $\Phi_{s}(p) \in \mathscr{D}_{1} \rightarrow 0$ as $p \rightarrow \infty$ then $\mathscr{D}_{1} \subseteq \mathscr{D}_{0}$. Moreover, for $\phi \in \mathscr{D}_{1}, \hat{T}_{0}$ coincides with Eq. 24] 29]. Using Eq. [18] and $\hat{H}(p)=i \hbar$, the eigenrelation

$$
\hat{H}(p)\left|\Phi_{s}(p)\right\rangle=\lambda\left|\Phi_{s}(p)\right\rangle
$$

is obtained. In order to find the expectation value for $\hat{H}$ we take the complex conjugate of Eq. 28], set $\hbar=1$, multiply by the eigenfunction $\Phi_{s}(p)$, and then integrate over $p$ to obtain

$$
\int_{-\infty}^{\infty}\left(i \frac{e^{-\frac{1}{2} i \pi s}(\operatorname{sgn}(p)+1) \sin (\pi s) \Gamma(1-s)|p|^{s-1}}{2 \pi^{3 / 2}}\right)^{*}\left(\frac{e^{-\frac{1}{2} i \pi s}(\operatorname{sgn}(p)+1) \sin (\pi s) \Gamma(1-s)|p|^{s-1}}{2 \pi^{3 / 2}}\right) d p=\lambda^{*}\|\Phi\|^{2},
$$

where $\lambda$ is the eigenvalue. It can be seen that the integral on the LHS vanishes for $\sigma>1 / 2$. Hence, using Eq. 20) on the RHS, Eq. 29 is then rewritten as

$$
\lim _{\sigma \rightarrow 1 / 2} \lambda^{*}(-1)^{-2 \sigma}=-\lambda^{*},
$$

demonstrating that the eigenvalues in momentum space vanish along the critical line $\sigma=1 / 2$. As a result, it is our ambition to study the eigenvalues in configuration space. 
Definition 1. The Riemann zeta Schrödinger equation is

$$
-\hbar \partial_{s}\left|\Psi_{s}(x)\right\rangle=i\left[\hat{\Delta}^{-1} \hat{x} \hat{p} \hat{\Delta}+\hat{\Delta}^{-1} \hat{p} \hat{x} \hat{\Delta}\right]\left|\Psi_{s}(x)\right\rangle,
$$

where $\hat{\Delta}$ is given by Eq. (8), $\hat{x}=x, \hat{p}=-i \hbar \partial_{x}, \hbar=1, x \in \mathbb{R}^{+} \geq 1$ owing to the difference operator $\hat{\Delta}\left|\Psi_{s}(x)\right\rangle$, and $s \in \mathbb{C}$.

Upon inserting Eq. (7) into Eq. (31) for $x \in \mathbb{R}^{+}$, we obtain the symmetrized Riemann zeta Schrödinger equation, i.e.,

$$
\begin{aligned}
\partial_{s}\left|\phi_{s}(x)\right\rangle & =1 / 2\left(\partial_{\sigma}-i \partial_{t}\right)\left|\phi_{s}(x)\right\rangle \\
& =-\frac{2}{\hbar} \sqrt{x} \partial_{x} \sqrt{x}\left|\phi_{s}(x)\right\rangle .
\end{aligned}
$$

Theorem 2. The eigenvalues of $\hat{H}=-2 i \hbar \sqrt{x} \partial_{x} \sqrt{x}$ occur at $\sigma= \pm 1 / 2 \forall t \in \mathbb{C}$ for $\hbar=1$ and $\left|\phi_{s}(x)\right\rangle=x^{-s}$ when $\mathscr{D}_{\hat{H}} \rightarrow(-\infty,-1] \cup[1, \infty)$.

Proof. Let $\left|\phi_{s}(x)\right\rangle$ be an eigenfunction of $\hat{H}$ with eigenvalue $t$, i.e.,

$$
\hat{H}\left|\phi_{s}(x)\right\rangle=t\left|\phi_{s}(x)\right\rangle \text {. }
$$

In order to find the expectation value for $\hat{H}$ we take the complex conjugate of Eq. 33 , multiply by the eigenfunction, and then integrate over $x \in[1, \infty),[-1,-\infty)$ to obtain

$$
\begin{aligned}
2 i \int_{-\infty}^{-1}\left(\sqrt{x} \partial_{x} \sqrt{x} \phi_{s}(x)\right)^{*} \phi_{s}(x) d x+2 i \int_{1}^{\infty}\left(\sqrt{x} \partial_{x} \sqrt{x} \phi_{s}(x)\right)^{*} \phi_{s}(x) d x & =t^{*} \int_{-\infty}^{-1} \phi_{s}^{*}(x) \phi_{s}(x) d x \\
& +t^{*} \int_{1}^{\infty} \phi_{s}^{*}(x) \phi_{s}(x) d x \\
& =t^{*}\|\phi\|^{2}
\end{aligned}
$$

Integrating by parts on the LHS then gives

$$
-2 i\left(\|\phi\|^{2}+\int_{-\infty}^{-1} \phi_{s}^{*}(x) x \frac{d}{d x} \phi_{s}(x) d x+\int_{1}^{\infty} \phi_{s}^{*}(x) x \frac{d}{d x} \phi_{s}(x) d x\right)=t^{*}\|\phi\|^{2} .
$$

Carrying out the integration on the LHS we obtain

$$
\frac{2 i(-1)^{-2 \sigma}\left((-1)^{2 \sigma}+1\right)(\sigma+i t)}{2 \sigma-1}=\left(t^{*}+2 i\right)\|\phi\|^{2} .
$$

Upon inserting Eq. 20] it can be seen that

$$
2 i(-1)^{-2 \sigma}\left((-1)^{2 \sigma}+1\right)(\sigma+i t)=\left(t^{*}+2 i\right)\left((-1)^{-2 \sigma}+1\right)
$$

Hence,

$$
\sigma= \pm \frac{1}{2} \forall t, t^{*}
$$

Taking the complex conjugate of Eq. 32 , setting $\hbar=1$, multiplying by the eigenfunction, and then integrating 
over $x \in[1, \infty),[-1,-\infty)$, it can be seen that

$$
\begin{aligned}
\frac{1}{2} \int_{-\infty}^{-1}\left(\partial_{\sigma} \phi_{s}(x)\right)^{*} \phi_{s}(x) d x+\frac{1}{2} \int_{1}^{\infty}\left(\partial_{\sigma} \phi_{s}(x)\right)^{*} \phi_{s}(x) d x & =-\frac{i}{2} \int_{-\infty}^{-1}\left(\partial_{t} \phi_{s}(x)\right)^{*} \phi_{s}(x) d x \\
& -\frac{i}{2} \int_{1}^{\infty}\left(\partial_{t} \phi_{s}(x)\right)^{*} \phi_{s}(x) d x \\
& -2 \int_{-\infty}^{-1}\left(\sqrt{x} \partial_{x} \sqrt{x} \phi_{s}(x)\right)^{*} \phi_{s}(x) d x \\
& -2 \int_{1}^{\infty}\left(\sqrt{x} \partial_{x} \sqrt{x} \phi_{s}(x)\right)^{*} \phi_{s}(x) d x
\end{aligned}
$$

Integrating by parts the last two terms on the RHS we obtain

$$
\begin{aligned}
\frac{1}{2} \int_{-\infty}^{-1}\left(\partial_{\sigma} \phi_{s}(x)\right)^{*} \phi_{s}(x) d x+\frac{1}{2} \int_{1}^{\infty}\left(\partial_{\sigma} \phi_{s}(x)\right)^{*} \phi_{s}(x) d x & =-\frac{i}{2} \int_{-\infty}^{-1}\left(\partial_{t} \phi_{s}(x)\right)^{*} \phi_{s}(x) d x \\
& -\frac{i}{2} \int_{1}^{\infty}\left(\partial_{t} \phi_{s}(x)\right)^{*} \phi_{s}(x) d x \\
& +2\|\phi\|^{2}+2 \int_{-\infty}^{-1} \phi_{s}^{*}(x) x \frac{d}{d x} \phi_{s}(x) d x \\
& +2 \int_{1}^{\infty} \phi_{s}^{*}(x) x \frac{d}{d x} \phi_{s}(x) d x
\end{aligned}
$$

Evaluating those integrals on LHS, we have

$$
\frac{1}{2} \int_{-\infty}^{-1}\left(\partial_{\sigma} \phi_{s}(x)\right)^{*} \phi_{s}(x) d x+\frac{1}{2} \int_{1}^{\infty}\left(\partial_{\sigma} \phi_{s}(x)\right)^{*} \phi_{s}(x) d x=-\frac{1}{2(1-2 \sigma)^{2}}+\frac{(-1)^{-2 \sigma}(-1+i \pi(1-2 \sigma))}{2(1-2 \sigma)^{2}}
$$

for $\sigma>1 / 2$. Evaluating those first two integrals on the RHS of Eq. 440, we have

$$
-\frac{i}{2} \int_{-\infty}^{-1}\left(\partial_{t} \phi_{s}(x)\right)^{*} \phi_{s}(x) d x-\frac{i}{2} \int_{1}^{\infty}\left(\partial_{t} \phi_{s}(x)\right)^{*} \phi_{s}(x) d x=\frac{1}{2(1-2 \sigma)^{2}}-\frac{i(-1)^{1-2 \sigma}(\pi(2 \sigma-1)-i)}{2(1-2 \sigma)^{2}},
$$

for $\sigma>1 / 2$. Evaluating those last two integrals on the RHS of Eq. 40, we have

$$
2 \int_{-\infty}^{-1} \phi_{s}^{*}(x) x \frac{d}{d x} \phi_{s}(x) d x+2 \int_{1}^{\infty} \phi_{s}^{*}(x) x \frac{d}{d x} \phi_{s}(x) d x=\frac{2(\sigma+i t)}{1-2 \sigma}+\frac{2(-1)^{1-2 \sigma}(\sigma+i t)}{2 \sigma-1},
$$

for $\sigma>1 / 2$. Finally, by using Eqs. (20) and 4143 in Eq. 40, it can be seen that

$$
\begin{aligned}
(-1)^{1-2 \sigma}\left(i \pi(2 \sigma-1)+(-1)^{2 \sigma}+1\right) & =(-1)^{-2 \sigma}\left(-8 \sigma^{2}-8 i \sigma t+(-1)^{2 \sigma+1}(4 \sigma(2 \sigma+2 i t-3)-4 i t+3)\right) \\
& +(-1)^{-2 \sigma}(2 i \pi \sigma+12 \sigma+4 i t-i \pi-3) .
\end{aligned}
$$

Hence, in the limit as $\sigma \rightarrow 1 / 2$, Eq. 44 is satisfied $\forall t, t^{*}$.

\section{A. Convergence}

Theorem 3. The eigenfunction $\phi_{s}(x)=x^{-s}$ of $\hat{H}=-2 i \hbar \sqrt{x} \partial_{x} \sqrt{x}$ normalizes at $x= \pm 1$.

Proof. In order to study convergent solutions to the Riemann zeta Schrödinger Eq. (32), it can be seen that upon inserting Eq. (7) into Eq. (32), we obtain

$$
\begin{aligned}
s & =\sigma+i t \\
& =\frac{1}{2}-\frac{\log (x)}{2} .
\end{aligned}
$$


Hence, at $x=1$ and letting $s=|s| e^{i \theta} \in \mathbb{C}$

$$
\theta=-i \log \left(\frac{1}{2|s|}\right)+2 \pi n,
$$

and

$$
|s|=\frac{e^{-i \theta}}{2},
$$

for $n \in \mathbb{Z}$, such that $\theta=\theta_{\Re}+i \theta_{\Im}$ like

$$
\theta_{\Re}=\arccos \left(\frac{1}{2|s|}\right)+2 \pi n,
$$

and

$$
\theta_{\Im}=2 \pi n
$$

At $x=-1$, Eq. 45 becomes

$$
\theta=-i \log \left(-\frac{i(\pi+i)}{2|s|}\right)+2 \pi n,
$$

and

$$
|s|=\frac{1}{2}\left(e^{-i \theta}-i \pi e^{-i \theta}\right),
$$

for $n \in \mathbb{Z}$, such that $\theta=\theta_{\Re}+i \theta_{\Im}$ like

$$
\theta_{\Re}=\arccos \left(\frac{1}{2|s|}\right)+2 \pi n,
$$

and

$$
\theta_{\Im}=\arcsin \left(-\frac{\pi}{2|s|}\right)+2 \pi n .
$$

In agreement with Eq. 21). This condition is required such that the density is normalized, i.e.,

$$
\begin{aligned}
\|\phi\|^{2} & =\sum_{m} \sum_{n} \hat{b}_{n}(s) \hat{b}_{m}^{\dagger}(s)\left\langle\phi_{m} \mid \phi_{n}\right\rangle \\
& =\sum_{n}\left|\hat{b}_{n}(s)\right|^{2} \\
& =i \pi .
\end{aligned}
$$

\section{B. Second Quantization}

Theorem 4. If all of the nontrivial zeros of $\zeta(s)=\left(1-2^{1-s}\right)^{-1} \sum_{n=1}^{\infty}(-1)^{n-1} n^{-s}$ occur at $s=1 / 2+i t_{n}$, then the numbers $t_{n}$ correspond to the eigenvalues (spectrum) of $\hat{H}=-2 i \hbar \sqrt{x} \partial_{x} \sqrt{x}$, and $n \in \mathbb{C}$, for the eigenstates $\left|\phi_{s}(x)\right\rangle=\hat{b}_{n}(s) x^{-\exp (-n+i n)|\sigma|}$.

Proof. In order to perform a second quantization [32, we can express the complex order parameter as a linear combination of basis states

$$
\left|\phi_{s}(x)\right\rangle=\sum_{n} \hat{b}_{n}(s)\left|\phi_{n}(x)\right\rangle
$$


where $s=|\sigma| \exp (i n) \in \mathbb{C},|\sigma| \in \mathbb{R}$ and $n$ is the complex phase. As such, using Eq. (10) we can rewrite Eq. 68) as

$$
\left|\phi_{n}(x)\right\rangle=\sum_{n} \hat{b}_{n}(s) x^{-\exp (-n+i n)|\sigma|},
$$

From Eq. 32 we find

$$
\hbar \frac{d}{d s} \hat{b}_{n}(s)=-t_{n} \hat{b}_{n}(s)
$$

We now find a Hamiltonian that yields Eq. (70) as the equation of motion. Hence, we take

$$
\left\langle\phi_{m}(x)|\hat{H}| \phi_{n}(x)\right\rangle=-2 \int_{1}^{\infty}\left\langle\phi_{m}(x)\left|\sqrt{x} \partial_{x} \sqrt{x}\right| \phi_{n}(x)\right\rangle d x-2 \int_{-\infty}^{-1}\left\langle\phi_{m}(x)\left|\sqrt{x} \partial_{x} \sqrt{x}\right| \phi_{n}(x)\right\rangle d x,
$$

as the expectation value. Upon substituting Eq. 69 into Eq. (71), we obtain the harmonic oscillator

$$
\begin{aligned}
\left\langle\phi_{m}(x)|\hat{H}| \phi_{n}(x)\right\rangle & =-2 \sum_{m} \sum_{n} \int_{1}^{\infty} x^{-\exp (-m-i m)|\sigma|} \sqrt{x} \partial_{x} \sqrt{x} x^{-\exp (-n+i n)|\sigma|} d x \\
& -2 \sum_{m} \sum_{n} \int_{-\infty}^{-1} x^{-\exp (-m-i m)|\sigma|} \sqrt{x} \partial_{x} \sqrt{x} x^{-\exp (-n+i n)|\sigma|} d x \\
& =-\sum_{m} \sum_{n} \hat{b}_{m}^{\dagger}(s) \hat{b}_{n}(s) \frac{e^{(1+i) m}\left(e^{n}-2 e^{i n}|\sigma|\right)(-1)^{1+|\sigma|}\left(-e^{(-1-i) m}-e^{(-1+i) n}\right)}{e^{n+(1+i) m}-|\sigma|\left(e^{n}+e^{(1+i) m+i n}\right)}|m, n\rangle \\
& +\sum_{m} \sum_{n} \hat{b}_{m}^{\dagger}(s) \hat{b}_{n}(s) \frac{e^{(1+i) m}\left(e^{n}-2 e^{i n}|\sigma|\right)}{e^{n+(1+i) m}-|\sigma|\left(e^{n}+e^{(1+i) m+i n}\right)}|m, n\rangle,
\end{aligned}
$$

for $|\sigma| \Re\left(e^{(-1-i) m}+e^{(-1+i) n}\right)>1$. Hence at $m=n$,

$$
\begin{aligned}
\left\langle\phi_{n}(x)|\hat{H}| \phi_{n}(x)\right\rangle & =-i \pi \sum_{n} \frac{e^{(1+i) n}(-1)^{1+\left(-e^{(-1-i) n}-e^{(-1+i) n}\right)|\sigma|}\left(e^{n}-2 e^{i n}|\sigma|\right)}{e^{(2+i) n}-\left(e^{n}+e^{(1+2 i) n}\right)|\sigma|}|n\rangle \\
& +i \pi \sum_{n}-\frac{e^{(1+i) n}\left(e^{n}-2 e^{i n}|\sigma|\right)}{e^{(2+i) n}-\left(e^{n}+e^{(1+2 i) n}\right)|\sigma|}|n\rangle
\end{aligned}
$$

where $|n\rangle=1,2,3, \ldots, \infty$. In accordance with Eq. [38), at $|\sigma|=1 / 2$,

$$
\begin{aligned}
\left\langle\phi_{n}(x)|\hat{H}| \phi_{n}(x)\right\rangle & =-i \pi \sum_{n=1}^{\infty} \frac{(-1)^{1+\frac{1}{2}\left(-e^{(-1-i) n}-e^{(-1+i) n}\right)} e^{(1+i) n}\left(e^{n}-e^{i n}\right)}{\frac{1}{2}\left(-e^{n}-e^{(1+2 i) n}\right)+e^{(2+i) n}}|n\rangle \\
& +i \pi \sum_{n=1}^{\infty} \frac{e^{(1+i) n}\left(e^{n}-e^{i n}\right)}{\frac{1}{2}\left(-e^{n}-e^{(1+2 i) n}\right)+e^{(2+i) n}}|n\rangle \\
& =-i \pi \sum_{n=1}^{\infty} \frac{2\left(e^{n}-e^{i n}\right) e^{i\left(n-\pi e^{-n} \cos (n)\right)}\left(1+e^{i \pi e^{-n} \cos (n)}\right)}{e^{2 i n}-2 e^{(1+i) n}+1}|n\rangle \\
& =2 \pi i \forall n .
\end{aligned}
$$

Theorem 5. If all of the nontrivial zeros of $\zeta(s)=\left(1-2^{1-s}\right)^{-1} \sum_{n=1}^{\infty}(-1)^{n-1} n^{-s}$ occur at $s=1 / 2+i t_{n}$, then the numbers $t_{n}$ correspond to the eigenvalues (spectrum) of $\hat{H}=-2 i \hbar \sqrt{x} \partial_{x} \sqrt{x}$, and $|n| \in \mathbb{Z} \subseteq \mathbb{R}$, for the eigenstates $\left|\phi_{s}(x)\right\rangle=x^{-n}$.

Proof. In order to perform a second quantization [32, we can express the complex order parameter as a linear combination of basis states

$$
\left|\phi_{s}(x)\right\rangle=\sum_{n} \hat{b}_{n}(s)\left|\phi_{n}(x)\right\rangle
$$


where $n \in \mathbb{Z} \subset \mathbb{R}$. As such, using Eq. 10 we can rewrite Eq. 68 as

$$
\left|\phi_{n}(x)\right\rangle=\sum_{n} \hat{b}_{n}(s) x^{-n}
$$

From Eq. (32) we find

$$
\hbar \frac{d}{d s} \hat{b}_{n}(s)=-t_{n} \hat{b}_{n}(s)
$$

We now find a Hamiltonian that yields Eq. 70 as the equation of motion. Hence, we take

$$
\left\langle\phi_{m}(x)|\hat{H}| \phi_{n}(x)\right\rangle=-2 \int_{1}^{\infty}\left\langle\phi_{m}(x)\left|\sqrt{x} \partial_{x} \sqrt{x}\right| \phi_{n}(x)\right\rangle d x-2 \int_{-\infty}^{-1}\left\langle\phi_{m}(x)\left|\sqrt{x} \partial_{x} \sqrt{x}\right| \phi_{n}(x)\right\rangle d x,
$$

as the expectation value. Upon substituting Eq. 69 into Eq. (71), we obtain the harmonic oscillator

$$
\begin{aligned}
\left\langle\phi_{m}(x)|\hat{H}| \phi_{n}(x)\right\rangle & =-2 \sum_{m} \sum_{n} \int_{1}^{\infty} x^{-m} \sqrt{x} \partial_{x} \sqrt{x} x^{-n} d x-2 \sum_{m} \sum_{n} \int_{-\infty}^{-1} x^{-m} \sqrt{x} \partial_{x} \sqrt{x} x^{-n} d x \\
& =\sum_{m} \sum_{n} \hat{b}_{m}^{\dagger}(s) \hat{b}_{n}(s) \frac{(2 n-1)(-1)^{-m-n}\left((-1)^{m+n}+1\right)}{m+n-1}|m, n\rangle \\
& =\sum_{m} \sum_{n} \frac{i \pi(2 n-1)(-1)^{-m-n}\left((-1)^{m+n}+1\right)}{m+n-1}|m, n\rangle
\end{aligned}
$$

for $\Re(m+n)>1$. Hence at $m=n$,

$$
\begin{aligned}
\left\langle\phi_{n}(x)|\hat{H}| \phi_{n}(x)\right\rangle & =\sum_{n} i \pi\left((-1)^{-2 n}+1\right)|n\rangle \\
& =2 \pi i \forall n,
\end{aligned}
$$

where $|n\rangle=1,2,3, \ldots, \infty$.

Theorem 6. If all of the nontrivial zeros of $\zeta(s)=\left(1-2^{1-s}\right)^{-1} \sum_{n=1}^{\infty}(-1)^{n-1} n^{-s}$ occur at $s=1 / 2+i t_{n}$, then the numbers $t_{n}$ correspond to the eigenvalues (spectrum) of $\hat{H}=-2 i \hbar \sqrt{x} \partial_{x} \sqrt{x}$, and $|n| \in \mathbb{Z} \subseteq \mathbb{R}$, for the eigenstates $\left|\phi_{s}(x)\right\rangle=\hat{b}_{n}(s) x^{-\exp (i n)|\sigma|}$.

Proof. In order to perform a second quantization [32, we can express the complex order parameter as a linear combination of basis states

$$
\left|\phi_{s}(x)\right\rangle=\sum_{n} \hat{b}_{n}(s)\left|\phi_{n}(x)\right\rangle
$$

where $s=|\sigma| \exp ($ in $) \in \mathbb{C}$, and $|\sigma| \in \mathbb{R}$. As such, using Eq. 10p we can rewrite Eq. (68) as

$$
\left|\phi_{n}(x)\right\rangle=\sum_{n} \hat{b}_{n}(s) x^{-\exp (i n)|\sigma|}
$$

From Eq. 32 we find

$$
\hbar \frac{d}{d s} \hat{b}_{n}(s)=-t_{n} \hat{b}_{n}(s)
$$

We now find a Hamiltonian that yields Eq. 70 as the equation of motion. Hence, we take

$$
\left\langle\phi_{m}(x)|\hat{H}| \phi_{n}(x)\right\rangle=-2 \int_{1}^{\infty}\left\langle\phi_{m}(x)\left|\sqrt{x} \partial_{x} \sqrt{x}\right| \phi_{n}(x)\right\rangle d x-2 \int_{-\infty}^{-1}\left\langle\phi_{m}(x)\left|\sqrt{x} \partial_{x} \sqrt{x}\right| \phi_{n}(x)\right\rangle d x,
$$

as the expectation value. 
Upon substituting Eq. 69 into Eq. (71), we obtain the harmonic oscillator

$$
\begin{aligned}
\left\langle\phi_{m}(x)|\hat{H}| \phi_{n}(x)\right\rangle & =-2 \sum_{m} \sum_{n} \int_{1}^{\infty} x^{-\exp (-i m)|\sigma|} \sqrt{x} \partial_{x} \sqrt{x} x^{-\exp (i n)|\sigma|} d x \\
& -2 \sum_{m} \sum_{n} \int_{-\infty}^{-1} x^{-\exp (-i m)|\sigma|} \sqrt{x} \partial_{x} \sqrt{x} x^{-\exp (i n)|\sigma|} d x \\
& =\sum_{m} \sum_{n} \hat{b}_{m}^{\dagger}(s) \hat{b}_{n}(s) \frac{e^{i m}\left(-1+2 e^{i n}|\sigma|\right)\left(1+(-1)^{-e^{-i m}|\sigma|\left(1+e^{i(m+n)}\right)}\right)}{|\sigma|\left(1+e^{i(m+n)}\right)-e^{i m}}|m\rangle, n,
\end{aligned}
$$

for $|\sigma| \Re\left(e^{-i m}+e^{i n}\right)>1$. Hence at $m=n$,

$$
\begin{aligned}
& \left\langle\phi_{n}(x)|\hat{H}| \phi_{n}(x)\right\rangle=\sum_{n} \frac{2 i \pi e^{2 i n}|\sigma|(-1)^{2|\sigma| \cos (n)} \cos (2 \pi|\sigma| \cos (n)+n)}{2|\sigma| \cos (n)-1}|n\rangle+\sum_{n} \frac{2 i \pi e^{2 i n}|\sigma| \cos (2 \pi|\sigma| \cos (n)+n)}{2|\sigma| \cos (n)-1}|n\rangle \\
& -\sum_{n} \frac{i \pi e^{i n}(-1)^{2|\sigma| \cos (n)} \cos (2 \pi|\sigma| \cos (n)+n)}{2|\sigma| \cos (n)-1}|n\rangle-\sum_{n} \frac{i \pi e^{i n} \cos (2 \pi|\sigma| \cos (n)+n)}{2|\sigma| \cos (n)-1}|n\rangle \\
& +\sum_{n} \frac{2 \pi e^{2 i n}|\sigma|(-1)^{2|\sigma| \cos (n)} \sin (2 \pi|\sigma| \cos (n)+n)}{2|\sigma| \cos (n)-1}|n\rangle+\sum_{n} \frac{2 \pi e^{2 i n}|\sigma| \sin (2 \pi|\sigma| \cos (n)+n)}{2|\sigma| \cos (n)-1}|n\rangle \\
& -\sum_{n} \frac{\pi e^{i n}(-1)^{2|\sigma| \cos (n)} \sin (2 \pi|\sigma| \cos (n)+n)}{2|\sigma| \cos (n)-1}|n\rangle-\sum_{n} \frac{\pi e^{i n} \sin (2 \pi|\sigma| \cos (n)+n)}{2|\sigma| \cos (n)-1}|n\rangle,
\end{aligned}
$$

where $|n\rangle=1,2,3, \ldots, \infty$. In accordance with Eq. (38), at $|\sigma|=1 / 2$,

$$
\begin{aligned}
\left\langle\phi_{n}(x)|\hat{H}| \phi_{n}(x)\right\rangle & =\pi \sum_{n=1}^{\infty}\left(1+e^{i \pi \cos (n)}\right)\left(\cot \left(\frac{n}{2}\right)+i\right)(\cos (\pi \cos (n))-i \sin (\pi \cos (n)))|n\rangle \\
& =0 \forall(2 n-1) \pi .
\end{aligned}
$$

Taking $\hat{b}_{n}(s)$ as an operator, and $\hat{b}_{n}^{\dagger}(s)$ as the adjoint, we obtain the usual properties:

$$
\begin{aligned}
& {\left[\hat{b}_{n}(s), \hat{b}_{m}(s)\right]=\left[\hat{b}_{n}^{\dagger}(s), \hat{b}_{m}^{\dagger}(s)\right]=0,} \\
& {\left[\hat{b}_{n}(s), \hat{b}_{m}^{\dagger}(s)\right]=\delta_{n m} .}
\end{aligned}
$$

From the analogous Heisenberg equations of motion,

$$
\begin{aligned}
-\hbar \frac{d}{d s} \sum_{n} \hat{b}_{n}(s) & =\left[\hat{b}_{n}(s), \hat{H}\right]_{-} \\
& =\sum_{m} E_{m}\left(\hat{b}_{n}(s) \hat{b}_{m}^{\dagger}(s) \hat{b}_{m}(s)-\hat{b}_{m}^{\dagger}(s) \hat{b}_{m}(s) \hat{b}_{n}(s)\right) \\
& =\sum_{m} E_{m}\left(\delta_{n m} \hat{b}_{m}(s)-\hat{b}_{m}^{\dagger}(s) \hat{b}_{n}(s) \hat{b}_{m}(s)-\hat{b}_{m}^{\dagger}(s) \hat{b}_{m}(s) \hat{b}_{n}(s)\right) \\
& =\sum_{m} E_{m}\left(\delta_{n m} \hat{b}_{m}(s)+\hat{b}_{m}^{\dagger}(s) \hat{b}_{m}(s) \hat{b}_{n}(s)-\hat{b}_{m}^{\dagger}(s) \hat{b}_{m}(s) \hat{b}_{n}(s)\right) \\
& =\sum_{n} \hat{b}_{m}^{\dagger}(s) \hat{b}_{n}(s) t_{n} .
\end{aligned}
$$

The eigenvalues of $\hat{H}$ are then

$$
\left\langle\phi_{n}(x)|\hat{H}| \phi_{n}(x)\right\rangle=\sum_{n} t_{n}|n\rangle
$$

However, since $t_{n}=i\left(\sigma_{n}-1 / 2\right)$ at the normalization constraint $x=1$, we can rewrite Eq. (77) as

$$
\left\langle\phi_{s}(x)|\hat{H}| \phi_{s}(x)\right\rangle=i \sum_{n}\left(\sigma_{n}-\frac{1}{2}\right)|n\rangle \text {. }
$$


From Eq. 76 it can be seen that

$$
\begin{aligned}
-\hbar \frac{d}{d s} \hat{b}_{n} & =i\left(\sigma_{n}-\frac{1}{2}\right) \hat{b}_{n} \\
-\hbar \frac{d}{d s} \hat{b}_{m}^{\dagger} & =-i\left(\sigma_{m}-\frac{1}{2}\right) \hat{b}_{m}^{\dagger} .
\end{aligned}
$$

Finally, upon setting $t_{n}=i\left(\sigma_{n}-1 / 2\right)$ and $t_{m}=-i\left(\sigma_{m}-1 / 2\right)$, we obtain

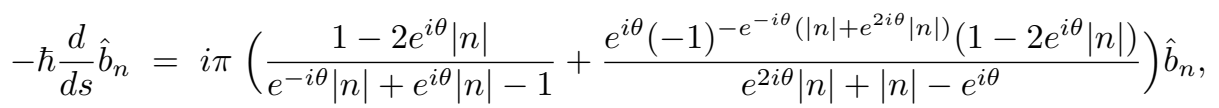

$$
\begin{aligned}
& -\hbar \frac{d}{d s} \hat{b}_{m}^{\dagger}=-i \pi\left(\frac{1-2 e^{i \theta}|m|}{e^{-i \theta}|m|+e^{i \theta}|m|-1}+\frac{e^{i \theta}(-1)^{-e^{-i \theta}\left(|m|+e^{2 i \theta}|m|\right)}\left(1-2 e^{i \theta}|m|\right)}{e^{2 i \theta}|m|+|m|-e^{i \theta}}\right) \hat{b}_{m}^{\dagger} .
\end{aligned}
$$

Eq. (76) is in agreement with Eq. 80, and can be solved using the Wirtinger derivatives.

Remark. Theorem 6 implies the Riemann hypothesis, as the spectrum of a Hermitian operator consists of real numbers as seen in Theorem 2.

Theorem 7. The densely defined operator $\hat{H}=-2 \sqrt{x} \partial_{x} \sqrt{x}$ on the Hilbert space $\mathscr{H}=L^{2}[1, \infty)$ is non-symmetric (non-Hermitian) [31] for the eigenstates $\phi_{n}(x)=\hat{b}_{n} x^{-\exp (-n+i n)|\sigma|}$ on $x \in(-\infty,-1] \cup[1, \infty)$ when $|\sigma|=1 / 2$.

Proof. By taking the inner products

$$
\begin{aligned}
\left(\hat{H} \phi^{*}, \phi\right) & =-2 i \pi \sum_{n=1}^{\infty} \int_{-\infty}^{-1} x^{-\exp (-n+i n)|\sigma|} \sqrt{x} \partial_{x} \sqrt{x} x^{-\exp (-n-i n)|\sigma|} d x \\
& -2 i \pi \sum_{n=1}^{\infty} \int_{1}^{\infty} x^{-\exp (-n+i n)|\sigma|} \sqrt{x} \partial_{x} \sqrt{x} x^{-\exp (-n-i n)|\sigma|} d x \\
& =\frac{i \pi\left(-2|\sigma|+e^{(1+i) n}\right)(-1)^{-2 e^{-n}|\sigma| \cos (n)}\left((-1)^{2 e^{-n}|\sigma| \cos (n)}+1\right)}{e^{(1+i) n}-\left(1+e^{2 i n}\right)|\sigma|},
\end{aligned}
$$

for $2|\sigma| \Re\left(e^{-n} \cos (n)\right)>1$, and

$$
\begin{aligned}
\left(\phi^{*}, \hat{H} \phi\right) & =-2 i \pi \sum_{n=1}^{\infty} \int_{-\infty}^{-1} x^{-\exp (-n-i n)|\sigma|} \sqrt{x} \partial_{x} \sqrt{x} x^{-\exp (-n+i n)|\sigma|} d x \\
& -2 i \pi \sum_{n=1}^{\infty} \int_{1}^{\infty} x^{-\exp (-n-i n)|\sigma|} \sqrt{x} \partial_{x} \sqrt{x} x^{-\exp (-n+i n)|\sigma|} d x \\
& =i \pi\left(\frac{\left(e^{n}-2 e^{i n}|\sigma|\right)(-1)^{-2 e^{-n}|\sigma| \cos (n)}}{e^{n}-2|\sigma| \cos (n)}+\frac{\left(-1+e^{2 i n}\right) \sigma}{e^{2 i n} \sigma-e^{(1+i) n} \operatorname{sgn}(\sigma)+\sigma}+1\right)
\end{aligned}
$$

for $2|\sigma| \Re\left(e^{-n} \cos (n)\right)>1$. It can be seen that

$$
\lim _{\sigma \rightarrow \frac{1}{2}}\left(\hat{H} \phi^{*}, \phi\right)=\frac{i \pi\left(-1+e^{(1+i) n}\right)(-1)^{-e^{-n} \cos (n)}\left((-1)^{e^{-n} \cos (n)}+1\right)}{\frac{1}{2}\left(-1-e^{2 i n}\right)+e^{(1+i) n}}
$$

and

$$
\lim _{\sigma \rightarrow \frac{1}{2}}\left(\phi^{*}, \hat{H} \phi\right)=\frac{i \pi\left(e^{n}-e^{i n}\right)(-1)^{-e^{-n} \cos (n)\left(1+i^{2 e^{-n} \cos (n)}\right)}}{e^{n}-\cos (n)}
$$

$\forall \phi, \phi^{*} \in \mathscr{D}(\hat{H})$.

Theorem 8. The densely defined operator $\hat{H}=-2 \sqrt{x} \partial_{x} \sqrt{x}$ on the Hilbert space $\mathscr{H}=L^{2}[1, \infty)$ is symmetric (Hermitian) [31] for the eigenstates $\phi_{n}(x)=\hat{b}_{n} x^{-\exp (i n)|\sigma|}$ on $x \in(-\infty,-1] \cup[1, \infty)$ when $|\sigma|=1 / 2$. 
Proof. By taking the inner products

$$
\begin{aligned}
\left(\hat{H} \phi^{*}, \phi\right) & =-2 i \pi \sum_{n=1}^{\infty} \int_{-\infty}^{-1} x^{-\exp (i n)|\sigma|} \sqrt{x} \partial_{x} \sqrt{x} x^{-\exp (-i n)|\sigma|} d x \\
& -2 i \pi \sum_{n=1}^{\infty} \int_{1}^{\infty} x^{-\exp (i n)|\sigma|} \sqrt{x} \partial_{x} \sqrt{x} x^{-\exp (-i n)|\sigma|} d x \\
& =-\sum_{n=1}^{\infty} \frac{2 \pi e^{-i n}\left(-2|\sigma|+e^{i n}\right) \cos ^{2}(\pi|\sigma| \cos (n))(\tan (\pi|\sigma| \cos (n))+i)}{2|\sigma| \cos (n)-1}
\end{aligned}
$$

for $2|\sigma| \Re(\cos (n))>1$, and

$$
\begin{aligned}
\left(\phi^{*}, \hat{H} \phi\right) & =-2 i \pi \sum_{n=1}^{\infty} \int_{-\infty}^{-1} x^{-\exp (-i n)|\sigma|} \sqrt{x} \partial_{x} \sqrt{x} x^{-\exp (i n)|\sigma|} d x \\
& -2 i \pi \sum_{n=1}^{\infty} \int_{1}^{\infty} x^{-\exp (-i n)|\sigma|} \sqrt{x} \partial_{x} \sqrt{x} x^{-\exp (i n)|\sigma|} d x \\
& =\frac{\pi\left(-1+2 e^{i n}|\sigma|\right)\left(\sin (2 \pi|\sigma| \cos (n))+2 i \cos ^{2}(\pi|\sigma| \cos (n))\right)}{2|\sigma| \cos (n)-1}
\end{aligned}
$$

for $2|\sigma| \Re(\cos (n))>1$. It can be seen that

$$
\lim _{\sigma \rightarrow \frac{1}{2}}\left(\hat{H} \phi^{*}, \phi\right)=-\frac{2 \pi e^{-i n}\left(-1+e^{i n}\right) \cos ^{2}\left(\frac{1}{2} \pi \cos (n)\right)\left(\tan \left(\frac{1}{2} \pi \cos (n)\right)+i\right)}{\cos (n)-1},
$$

and

$$
\lim _{\sigma \rightarrow \frac{1}{2}}\left(\phi^{*}, \hat{H} \phi\right)=\frac{\pi\left(-1+e^{i n}\right)\left(\sin (\pi \cos (n))+2 i \cos ^{2}\left(\frac{1}{2} \pi \cos (n)\right)\right)}{\cos (n)-1},
$$

$\forall \phi, \phi^{*} \in \mathscr{D}(\hat{H})$. Finally,

$$
\lim _{\sigma \rightarrow \frac{1}{2}}\left(\hat{H} \phi^{*}, \phi\right)=\lim _{\sigma \rightarrow \frac{1}{2}}\left(\phi^{*}, \hat{H} \phi\right), \forall 2 \pi n
$$

Theorem 9. The densely defined operator $\hat{H}=-2 \sqrt{x} \partial_{x} \sqrt{x}$ on the Hilbert space $\mathscr{H}=L^{2}[1, \infty)$ is holomorphic for the eigenstates $\phi_{\theta}(x)=\hat{b}_{\theta} x^{-|\sigma| \exp (i \theta)}$ on $x \in(-\infty,-1] \cup[1, \infty)$.

Proof. By taking the inner products

$$
\begin{aligned}
\left(\hat{H} \phi^{*}, \phi\right) & =-2 i \pi \sum_{\theta=1}^{\infty} \int_{-\infty}^{-1} x^{-|\sigma| \exp (i \theta)} \sqrt{x} \partial_{x} \sqrt{x} x^{-|\sigma| \exp (-i \theta)} d x-2 i \pi \sum_{\theta=1}^{\infty} \int_{1}^{\infty} x^{-|\sigma| \exp (i \theta)} \sqrt{x} \partial_{x} \sqrt{x} x^{-|\sigma| \exp (-i \theta)} d x \\
& =-2 i \pi \sum_{\theta=1}^{\infty}\left(\frac{1}{2}-\exp (-i \theta)|\sigma|\right) \int_{-\infty}^{-1} x^{-2|\sigma| \cos (\theta)} d x-2 i \pi \sum_{\theta=1}^{\infty}\left(\frac{1}{2}-\exp (-i \theta)|\sigma|\right) \int_{1}^{\infty} x^{-2|\sigma| \cos (\theta)} d x \\
& =-2 i \pi \sum_{\theta=1}^{\infty}\left(\frac{1}{2}-\exp (-i \theta)|\sigma|\right) \frac{(-1)^{-2|\sigma| \cos (\theta)}}{2|\sigma| \cos (\theta)-1}-2 i \pi \sum_{\theta=1}^{\infty}\left(\frac{1}{2}-\exp (-i \theta)|\sigma|\right) \frac{1}{2|\sigma| \cos (\theta)-1} \\
& =-2 i \pi \sum_{\theta=1}^{\infty}\left(\frac{1}{2}-\exp (-i \theta)|\sigma|\right) \frac{(-1)^{-2|\sigma| \cos (\theta)}+1}{2|\sigma| \cos (\theta)-1}
\end{aligned}
$$

and 


$$
\begin{aligned}
\left(\phi^{*}, \hat{H} \phi\right) & =-2 i \pi \sum_{\theta=1}^{\infty} \int_{-\infty}^{-1} x^{-|\sigma| \exp (-i \theta)} \sqrt{x} \partial_{x} \sqrt{x} x^{-|\sigma| \exp (i \theta)} d x-2 i \pi \sum_{\theta=1}^{\infty} \int_{1}^{\infty} x^{-|\sigma| \exp (-i \theta)} \sqrt{x} \partial_{x} \sqrt{x} x^{-|\sigma| \exp (i \theta)} d x \\
& =-2 i \pi \sum_{\theta=1}^{\infty}\left(\frac{1}{2}-\exp (i \theta)|\sigma|\right) \int_{-\infty}^{-1} x^{-2|\sigma| \cos (\theta)} d x-2 i \pi \sum_{\theta=1}^{\infty}\left(\frac{1}{2}-\exp (i \theta)|\sigma|\right) \int_{1}^{\infty} x^{-2|\sigma| \cos (\theta)} d x \\
& =-2 i \pi \sum_{\theta=1}^{\infty}\left(\frac{1}{2}-\exp (i \theta)|\sigma|\right) \frac{(-1)^{-2|\sigma| \cos (\theta)}}{2|\sigma| \cos (\theta)-1}-2 i \pi \sum_{\theta=1}^{\infty}\left(\frac{1}{2}-\exp (i \theta)|\sigma|\right) \frac{1}{2|\sigma| \cos (\theta)-1} \\
& =-2 i \pi \sum_{\theta=1}^{\infty}\left(\frac{1}{2}-\exp (i \theta)|\sigma|\right) \frac{(-1)^{-2|\sigma| \cos (\theta)}+1}{2|\sigma| \cos (\theta)-1}
\end{aligned}
$$

it can be seen that

$$
\oint_{C}\left(\hat{H} \phi^{*}, \phi\right) d \theta=\oint_{C}\left(\phi^{*}, \hat{H} \phi\right) d \theta=0
$$

$\forall \phi, \phi^{*} \in \mathscr{D}(\hat{H})$. Here, it is useful to implement the following identities:

$$
\cos (\theta)=\cos \left(\theta_{\Re}\right) \cosh \left(\theta_{\Im}\right)-i \sin \left(\theta_{\Re}\right) \sinh \left(\theta_{\Im}\right),
$$

and

$$
\exp ( \pm i \theta)=\exp \left(-\theta_{\Im}\right) \cos \left(\theta_{\Re}\right) \pm i \exp \left(-\theta_{\Im}\right) \sin \left(\theta_{\Re}\right) .
$$

where $\theta=\theta_{\Re}+i \theta_{\Im}$. The path $C$ is then parametrized as follows:

$$
\theta(q)=|\theta| \cos (q)+i|\theta| \sin (q),
$$

with $0 \leq q \leq 2 \pi$, whilst $f(\theta)$ is given by Eq. (50), and the real and imaginary parts of Eq. (50) are given by Eqs. 52 and (53), and

$$
|\theta|=\sqrt{\alpha^{2}+\beta^{2}}
$$

where

$$
\alpha=2|\sigma| \cos \left(\theta_{\Re}\right) \cosh \left(\theta_{\Im}\right)-1,
$$

and

$$
\beta=-2|\sigma| \sin \left(\theta_{\Re}\right) \sinh \left(\theta_{\Im}\right) .
$$

By performing the parametrization of the path $C$, the real and imaginary parts of $\left(\phi^{*}, \hat{H} \phi\right)$ are then

$$
\begin{aligned}
\Re\left(\phi^{*}, \hat{H} \phi\right) & =\frac{\pi}{|\theta|^{2}}(-(1+\alpha) \exp (\pi \beta) \sin (\pi(1+\alpha))-\beta \exp (\pi \beta) \cos (\pi(1+\alpha)) \\
& +\exp (\pi \beta) \sin (\pi(1+\alpha))-\beta+2|\sigma|(\alpha+1) \exp \left(-\theta_{\Im}\right) \cos \left(\theta_{\Re}\right) \exp (\pi \beta) \sin (\pi(1+\alpha)) \\
& +2 \beta|\sigma| \exp \left(-\theta_{\Im}\right) \cos \left(\theta_{\Re}\right) \exp (\pi \beta) \cos (\pi(1+\alpha))-2|\sigma| \exp \left(-\theta_{\Im}\right) \cos \left(\theta_{\Re}\right) \exp (\pi \beta) \sin (\pi(1+\alpha)) \\
& +2|\sigma| \beta \exp \left(-\theta_{\Im}\right) \cos \left(\theta_{\Re}\right)-2|\sigma|(1+\alpha) \exp \left(-\theta_{\Im}\right) \sin \left(\theta_{\Re}\right) \exp (\pi \beta) \cos (\pi(1+\alpha)) \\
& +2|\sigma| \beta \exp \left(-\theta_{\Im}\right) \sin \left(\theta_{\Re}\right) \exp (\pi \beta) \sin (\pi(1+\alpha)) \\
& +2|\sigma| \exp \left(-\theta_{\Im}\right) \sin \left(\theta_{\Re}\right) \exp (\pi \beta) \cos (\pi(1+\alpha))-2|\sigma|(\alpha+1) \exp \left(-\theta_{\Im}\right) \sin \left(\theta_{\Re}\right) \\
& \left.+2|\sigma| \exp \left(-\theta_{\Im}\right) \sin \left(\theta_{\Re}\right)\right)
\end{aligned}
$$

and 


$$
\begin{aligned}
\Im\left(\phi^{*}, \hat{H} \phi\right) & =\frac{\pi}{|\theta|^{2}}(-(1+\alpha) \exp (\pi \beta) \cos (\pi(1+\alpha))+\beta \exp (\pi \beta) \sin (\pi(1+\alpha)) \\
& +\exp (\pi \beta) \cos (\pi(1+\alpha))-\alpha \\
& +2|\sigma|(1+\alpha) \cos \left(\theta_{\Re}\right) \exp \left(-\theta_{\Im}\right) \exp (\pi \beta) \cos (\pi(1+\alpha)) \\
& -2|\sigma| \beta \cos \left(\theta_{\Re}\right) \exp \left(-\theta_{\Im}\right) \exp (\pi \beta) \sin (\pi(1+\alpha)) \\
& -2|\sigma| \cos \left(\theta_{\Re}\right) \exp \left(-\theta_{\Im}\right) \exp (\pi \beta) \cos (\pi(1+\alpha))+2|\sigma|(\alpha+1) \cos \left(\theta_{\Re}\right) \exp \left(-\theta_{\Im}\right) \\
& -2|\sigma| \cos \left(\theta_{\Re}\right) \exp \left(-\theta_{\Im}\right)+2|\sigma| \beta \sin \left(\theta_{\Re}\right) \exp \left(-\theta_{\Im}\right) \\
& +2|\sigma|(\alpha+1) \sin \left(\theta_{\Re}\right) \exp \left(-\theta_{\Im}\right) \exp (\pi \beta) \sin (\pi(1+\alpha)) \\
& +2|\sigma| \beta \sin \left(\theta_{\Re}\right) \exp \left(-\theta_{\Im}\right) \exp (\pi \beta) \cos (\pi(1+\alpha)) \\
& \left.-2|\sigma| \sin \left(\theta_{\Re}\right) \exp \left(-\theta_{\Im}\right) \exp (\pi \beta) \sin (\pi(1+\alpha))\right)
\end{aligned}
$$

where

$$
\begin{aligned}
& \Re\left(\phi^{*}, \hat{H} \phi\right)=\frac{-2 \pi|\sigma| \cos \left(\theta_{\Re}\right) \cosh \left(\theta_{\Im}\right) \exp \left(-2 \pi \sigma \sin \left(\theta_{\Re}\right) \sinh \left(\theta_{\Im}\right)\right) \sin \left(2 \pi \sigma \cos \left(\theta_{\Re}\right) \cosh \left(\theta_{\Im}\right)\right)}{1-4|\sigma| \cos \left(\theta_{\Re}\right) \cosh \left(\theta_{\Im}\right)+2 \sigma^{2}\left(\cos \left(2 \theta_{\Re}\right)+\cosh \left(2 \theta_{\Im}\right)\right)} \\
& +\frac{2 \pi|\sigma| \sin \left(\theta_{\Re}\right) \sinh \left(\theta_{\Im}\right) \exp \left(-2 \pi \sigma \sin \left(\theta_{\Re}\right) \sinh \left(\theta_{\Im}\right)\right) \cos \left(2 \pi \sigma \cos \left(\theta_{\Re}\right) \cosh \left(\theta_{\Im}\right)\right)}{1-4|\sigma| \cos \left(\theta_{\Re}\right) \cosh \left(\theta_{\Im}\right)+2 \sigma^{2}\left(\cos \left(2 \theta_{\Re}\right)+\cosh \left(2 \theta_{\Im}\right)\right)} \\
& +\frac{\pi \exp \left(-2 \pi \sigma \sin \left(\theta_{\Re}\right) \sinh \left(\theta_{\Im}\right)\right) \sin \left(2 \pi \sigma \cos \left(\theta_{\Re}\right) \cosh \left(\theta_{\Im}\right)\right)}{1-4|\sigma| \cos \left(\theta_{\Re}\right) \cosh \left(\theta_{\Im}\right)+2 \sigma^{2}\left(\cos \left(2 \theta_{\Re}\right)+\cosh \left(2 \theta_{\Im}\right)\right)} \\
& +\frac{2 \pi|\sigma| \sin \left(\theta_{\Re}\right) \sinh \left(\theta_{\Im}\right)}{1-4|\sigma| \cos \left(\theta_{\Re}\right) \cosh \left(\theta_{\Im}\right)+2 \sigma^{2}\left(\cos \left(2 \theta_{\Re}\right)+\cosh \left(2 \theta_{\Im}\right)\right)} \\
& +\frac{4 \pi|\sigma|^{2} \cos ^{2}\left(\theta_{\Re}\right) \cosh \left(\theta_{\Im}\right) \exp \left(-\theta_{\Im}\right) \exp \left(-2 \pi \sigma \sin \left(\theta_{\Re}\right) \sinh \left(\theta_{\Im}\right)\right) \sin \left(2 \pi \sigma \cos \left(\theta_{\Re}\right) \cosh \left(\theta_{\Im}\right)\right)}{1-4|\sigma| \cos \left(\theta_{\Re}\right) \cosh \left(\theta_{\Im}\right)+2 \sigma^{2}\left(\cos \left(2 \theta_{\Re}\right)+\cosh \left(2 \theta_{\Im}\right)\right)} \\
& +\frac{-4 \pi|\sigma|^{2} \cos \left(\theta_{\Re}\right) \sin \left(\theta_{\Re}\right) \sinh \left(\theta_{\Im}\right) \exp \left(-\theta_{\Im}\right) \exp \left(-2 \pi \sigma \sin \left(\theta_{\Re}\right) \sinh \left(\theta_{\Im}\right)\right) \cos \left(2 \pi \sigma \cos \left(\theta_{\Re}\right) \cosh \left(\theta_{\Im}\right)\right)}{1-4|\sigma| \cos \left(\theta_{\Re}\right) \cosh \left(\theta_{\Im}\right)+2 \sigma^{2}\left(\cos \left(2 \theta_{\Re}\right)+\cosh \left(2 \theta_{\Im}\right)\right)} \\
& +\frac{-2 \pi|\sigma| \cos \left(\theta_{\Re}\right) \exp \left(-\theta_{\Im}\right) \exp \left(-2 \pi \sigma \sin \left(\theta_{\Re}\right) \sinh \left(\theta_{\Im}\right)\right) \sin \left(2 \pi \sigma \cos \left(\theta_{\Re}\right) \cosh \left(\theta_{\Im}\right)\right)}{1-4|\sigma| \cos \left(\theta_{\Re}\right) \cosh \left(\theta_{\Im}\right)+2 \sigma^{2}\left(\cos \left(2 \theta_{\Re}\right)+\cosh \left(2 \theta_{\Im}\right)\right)} \\
& +\frac{-4 \pi|\sigma|^{2} \exp \left(-\theta_{\Im}\right) \cos \left(\theta_{\Re}\right) \sin \left(\theta_{\Re}\right) \sinh \left(\theta_{\Im}\right)}{1-4|\sigma| \cos \left(\theta_{\Re}\right) \cosh \left(\theta_{\Im}\right)+2 \sigma^{2}\left(\cos \left(2 \theta_{\Re}\right)+\cosh \left(2 \theta_{\Im}\right)\right)} \\
& +\frac{-2 \pi|\sigma| \cos \left(\theta_{\Re}\right) \exp \left(-\theta_{\Im}\right) \exp \left(-2 \pi \sigma \sin \left(\theta_{\Re}\right) \sinh \left(\theta_{\Im}\right)\right) \sin \left(2 \pi \sigma \cos \left(\theta_{\Re}\right) \cosh \left(\theta_{\Im}\right)\right)}{1-4|\sigma| \cos \left(\theta_{\Re}\right) \cosh \left(\theta_{\Im}\right)+2 \sigma^{2}\left(\cos \left(2 \theta_{\Re}\right)+\cosh \left(2 \theta_{\Im}\right)\right)} \\
& +\frac{-4 \pi|\sigma|^{2} \exp \left(-\theta_{\Im}\right) \cos \left(\theta_{\Re}\right) \sin \left(\theta_{\Re}\right) \cosh \left(\theta_{\Im}\right) \exp \left(-2 \pi \sigma \sin \left(\theta_{\Re}\right) \sinh \left(\theta_{\Im}\right)\right) \cos \left(2 \pi \sigma \cos \left(\theta_{\Re}\right) \cosh \left(\theta_{\Im}\right)\right)}{1-4|\sigma| \cos \left(\theta_{\Re}\right) \cosh \left(\theta_{\Im}\right)+2 \sigma^{2}\left(\cos \left(2 \theta_{\Re}\right)+\cosh \left(2 \theta_{\Im}\right)\right)} \\
& +\frac{-4 \pi|\sigma|^{2} \exp \left(-\theta_{\Im}\right) \sin ^{2}\left(\theta_{\Re}\right) \sinh \left(\theta_{\Im}\right) \exp \left(-2 \pi \sigma \sin \left(\theta_{\Re}\right) \sinh \left(\theta_{\Im}\right)\right) \sin \left(2 \pi \sigma \cos \left(\theta_{\Re}\right) \cosh \left(\theta_{\Im}\right)\right)}{1-4|\sigma| \cos \left(\theta_{\Re}\right) \cosh \left(\theta_{\Im}\right)+2 \sigma^{2}\left(\cos \left(2 \theta_{\Re}\right)+\cosh \left(2 \theta_{\Im}\right)\right)}
\end{aligned}
$$




$$
\begin{aligned}
& +\frac{2 \pi|\sigma| \exp \left(-\theta_{\Im}\right) \sin \left(\theta_{\Re}\right) \exp \left(-2 \pi \sigma \sin \left(\theta_{\Re}\right) \sinh \left(\theta_{\Im}\right)\right) \cos \left(2 \pi \sigma \cos \left(\theta_{\Re}\right) \cosh \left(\theta_{\Im}\right)\right)}{1-4|\sigma| \cos \left(\theta_{\Re}\right) \cosh \left(\theta_{\Im}\right)+2 \sigma^{2}\left(\cos \left(2 \theta_{\Re}\right)+\cosh \left(2 \theta_{\Im}\right)\right)} \\
& +\frac{-4 \pi|\sigma|^{2} \exp \left(-\theta_{\Im}\right) \sin \left(\theta_{\Re}\right) \cos \left(\theta_{\Re}\right) \cosh \left(\theta_{\Im}\right)}{1-4|\sigma| \cos \left(\theta_{\Re}\right) \cosh \left(\theta_{\Im}\right)+2 \sigma^{2}\left(\cos \left(2 \theta_{\Re}\right)+\cosh \left(2 \theta_{\Im}\right)\right)} \\
& +\frac{2 \pi|\sigma| \exp \left(-\theta_{\Im}\right) \sin \left(\theta_{\Re}\right)}{1-4|\sigma| \cos \left(\theta_{\Re}\right) \cosh \left(\theta_{\Im}\right)+2 \sigma^{2}\left(\cos \left(2 \theta_{\Re}\right)+\cosh \left(2 \theta_{\Im}\right)\right)}
\end{aligned}
$$

and

$$
\begin{aligned}
& \Im\left(\phi^{*}, \hat{H} \phi\right)=\frac{-2 \pi|\sigma| \cos \left(\theta_{\Re}\right) \cosh \left(\theta_{\Im}\right) \exp \left(-2 \pi \sigma \sin \left(\theta_{\Re}\right) \sinh \left(\theta_{\Im}\right)\right) \cos \left(2 \pi \sigma \cos \left(\theta_{\Re}\right) \cosh \left(\theta_{\Im}\right)\right)}{1-4|\sigma| \cos \left(\theta_{\Re}\right) \cosh \left(\theta_{\Im}\right)+2 \sigma^{2}\left(\cos \left(2 \theta_{\Re}\right)+\cosh \left(2 \theta_{\Im}\right)\right)} \\
& +\frac{-2 \pi|\sigma| \sin \left(\theta_{\Re}\right) \sinh \left(\theta_{\Im}\right) \exp \left(-2 \pi \sigma \sin \left(\theta_{\Re}\right) \sinh \left(\theta_{\Im}\right)\right) \sin \left(2 \pi \sigma \cos \left(\theta_{\Re}\right) \cosh \left(\theta_{\Im}\right)\right)}{1-4|\sigma| \cos \left(\theta_{\Re}\right) \cosh \left(\theta_{\Im}\right)+2 \sigma^{2}\left(\cos \left(2 \theta_{\Re}\right)+\cosh \left(2 \theta_{\Im}\right)\right)} \\
& +\frac{\pi \exp \left(-2 \pi \sigma \sin \left(\theta_{\Re}\right) \sinh \left(\theta_{\Im}\right)\right) \cos \left(2 \pi \sigma \cos \left(\theta_{\Re}\right) \cosh \left(\theta_{\Im}\right)\right)}{1-4|\sigma| \cos \left(\theta_{\Re}\right) \cosh \left(\theta_{\Im}\right)+2 \sigma^{2}\left(\cos \left(2 \theta_{\Re}\right)+\cosh \left(2 \theta_{\Im}\right)\right)} \\
& +\frac{-2 \pi|\sigma| \cos \left(\theta_{\Re}\right) \cosh \left(\theta_{\Im}\right)}{1-4|\sigma| \cos \left(\theta_{\Re}\right) \cosh \left(\theta_{\Im}\right)+2 \sigma^{2}\left(\cos \left(2 \theta_{\Re}\right)+\cosh \left(2 \theta_{\Im}\right)\right)} \\
& +\frac{\pi}{1-4|\sigma| \cos \left(\theta_{\Re}\right) \cosh \left(\theta_{\Im}\right)+2 \sigma^{2}\left(\cos \left(2 \theta_{\Re}\right)+\cosh \left(2 \theta_{\Im}\right)\right)} \\
& +\frac{4 \pi|\sigma|^{2} \cos ^{2}\left(\theta_{\Re}\right) \cosh \left(\theta_{\Im}\right) \exp \left(-\theta_{\Im}\right) \exp \left(-2 \pi \sigma \sin \left(\theta_{\Re}\right) \sinh \left(\theta_{\Im}\right)\right) \cos \left(2 \pi \sigma \cos \left(\theta_{\Re}\right) \cosh \left(\theta_{\Im}\right)\right)}{1-4|\sigma| \cos \left(\theta_{\Re}\right) \cosh \left(\theta_{\Im}\right)+2 \sigma^{2}\left(\cos \left(2 \theta_{\Re}\right)+\cosh \left(2 \theta_{\Im}\right)\right)} \\
& +\frac{4 \pi|\sigma|^{2} \sin \left(\theta_{\Re}\right) \sinh \left(\theta_{\Im}\right) \exp \left(-\theta_{\Im}\right) \cos \left(\theta_{\Re}\right) \exp \left(-2 \pi \sigma \sin \left(\theta_{\Re}\right) \sinh \left(\theta_{\Im}\right)\right) \sin \left(2 \pi \sigma \cos \left(\theta_{\Re}\right) \cosh \left(\theta_{\Im}\right)\right)}{1(\theta))} \\
& 1-4|\sigma| \cos \left(\theta_{\Re}\right) \cosh \left(\theta_{\Im}\right)+2 \sigma^{2}\left(\cos \left(2 \theta_{\Re}\right)+\cosh \left(2 \theta_{\Im}\right)\right) \\
& +\frac{-2 \pi|\sigma| \cos \left(\theta_{\Re}\right) \exp \left(-\theta_{\Im}\right) \exp \left(-2 \pi \sigma \sin \left(\theta_{\Re}\right) \sinh \left(\theta_{\Im}\right)\right) \cos \left(2 \pi \sigma \cos \left(\theta_{\Re}\right) \cosh \left(\theta_{\Im}\right)\right)}{1-4|\sigma| \cos \left(\theta_{\Re}\right) \cosh \left(\theta_{\Im}\right)+2 \sigma^{2}\left(\cos \left(2 \theta_{\Re}\right)+\cosh \left(2 \theta_{\Im}\right)\right)} \\
& +\frac{4 \pi|\sigma|^{2} \cos ^{2}\left(\theta_{\Re}\right) \cosh \left(\theta_{\Im}\right) \exp \left(-\theta_{\Im}\right)}{1-4|\sigma| \cos \left(\theta_{\Re}\right) \cosh \left(\theta_{\Im}\right)+2 \sigma^{2}\left(\cos \left(2 \theta_{\Re}\right)+\cosh \left(2 \theta_{\Im}\right)\right)} \\
& +\frac{-2 \pi|\sigma| \cos \left(\theta_{\Re}\right) \exp \left(-\theta_{\Im}\right)}{1-4|\sigma| \cos \left(\theta_{\Re}\right) \cosh \left(\theta_{\Im}\right)+2 \sigma^{2}\left(\cos \left(2 \theta_{\Re}\right)+\cosh \left(2 \theta_{\Im}\right)\right)} \\
& +\frac{4 \pi|\sigma|^{2} \cos \left(\theta_{\Re}\right) \sin \left(\theta_{\Re}\right) \cosh \left(\theta_{\Im}\right) \exp \left(-\theta_{\Im}\right) \exp \left(-2 \pi \sigma \sin \left(\theta_{\Re}\right) \sinh \left(\theta_{\Im}\right)\right) \sin \left(2 \pi \sigma \cos \left(\theta_{\Re}\right) \cosh \left(\theta_{\Im}\right)\right)}{1-4|\sigma| \cos \left(\theta_{\Re}\right) \cosh \left(\theta_{\Im}\right)+2 \sigma^{2}\left(\cos \left(2 \theta_{\Re}\right)+\cosh \left(2 \theta_{\Im}\right)\right)} \\
& +\frac{-4 \pi|\sigma|^{2} \sin ^{2}\left(\theta_{\Re}\right) \sinh \left(\theta_{\Im}\right) \exp \left(-\theta_{\Im}\right) \exp \left(-2 \pi \sigma \sin \left(\theta_{\Re}\right) \sinh \left(\theta_{\Im}\right)\right) \cos \left(2 \pi \sigma \cos \left(\theta_{\Re}\right) \cosh \left(\theta_{\Im}\right)\right)}{1-4|\sigma| \cos \left(\theta_{\Re}\right) \cosh \left(\theta_{\Im}\right)+2 \sigma^{2}\left(\cos \left(2 \theta_{\Re}\right)+\cosh \left(2 \theta_{\Im}\right)\right)} \\
& +\frac{-2 \pi|\sigma| \sin \left(\theta_{\Re}\right) \exp \left(-\theta_{\Im}\right) \exp \left(-2 \pi \sigma \sin \left(\theta_{\Re}\right) \sinh \left(\theta_{\Im}\right)\right) \sin \left(2 \pi \sigma \cos \left(\theta_{\Re}\right) \cosh \left(\theta_{\Im}\right)\right)}{1-4|\sigma| \cos \left(\theta_{\Re}\right) \cosh \left(\theta_{\Im}\right)+2 \sigma^{2}\left(\cos \left(2 \theta_{\Re}\right)+\cosh \left(2 \theta_{\Im}\right)\right)}
\end{aligned}
$$




$$
+\frac{-4 \pi|\sigma|^{2} \sin ^{2}\left(\theta_{\Re}\right) \sinh \left(\theta_{\Im}\right) \exp \left(-\theta_{\Im}\right)}{1-4|\sigma| \cos \left(\theta_{\Re}\right) \cosh \left(\theta_{\Im}\right)+2 \sigma^{2}\left(\cos \left(2 \theta_{\Re}\right)+\cosh \left(2 \theta_{\Im}\right)\right)} .
$$

Hence, from the relation

$$
\begin{aligned}
\oint_{C}\left(\phi^{*}, \hat{H} \phi\right) d \theta & =\oint_{C}\left(\Re\left(\phi^{*}, \hat{H} \phi\right)+i \Im\left(\phi^{*}, \hat{H} \phi\right)\right)\left(d \theta_{\Re}+i d \theta_{\Im}\right) \\
& =\oint_{C} \Re\left(\phi^{*}, \hat{H} \phi\right) d \theta_{\Re}-\oint_{C} \Im\left(\phi^{*}, \hat{H} \phi\right) d \theta_{\Im} \\
& +i \oint_{C} \Re\left(\phi^{*}, \hat{H} \phi\right) d \theta_{\Im}+i \oint_{C} \Im\left(\phi^{*}, \hat{H} \phi\right) d \theta_{\Re} \\
& =\int_{0}^{2 \pi} \Re\left(\phi^{*}, \hat{H} \phi\right) \frac{d \theta_{\Re}}{d q} d q-\int_{0}^{2 \pi} \Im\left(\phi^{*}, \hat{H} \phi\right) \frac{d \theta_{\Im}}{d q} d q \\
& +i \int_{0}^{2 \pi} \Re\left(\phi^{*}, \hat{H} \phi\right) \frac{d \theta_{\Im}}{d q} d q+i \int_{0}^{2 \pi} \Im\left(\phi^{*}, \hat{H} \phi\right) \frac{d \theta_{\Re}}{d q} d q
\end{aligned}
$$

at $|\theta|=1$, we obtain

$$
\begin{aligned}
& \int_{0}^{2 \pi} \Re\left(\phi^{*}, \hat{H} \phi\right) \frac{d \theta_{\Re}}{d q} d q=\frac{1}{2} \pi^{4}\left(2 \exp \left(-\theta_{\Im}\right) \cos \left(\theta_{\Re}\right)|\theta|-1\right), \\
& \int_{0}^{2 \pi} \Im\left(\phi^{*}, \hat{H} \phi\right) \frac{d \theta_{\Im}}{d q} d q=\frac{1}{2} \pi^{4}\left(2 \exp \left(-\theta_{\Im}\right) \cos \left(\theta_{\Re}\right)|\theta|-1\right), \\
& \int_{0}^{2 \pi} \Re\left(\phi^{*}, \hat{H} \phi\right) \frac{d \theta_{\Im}}{d q} d q=-\pi^{4} \exp \left(-\theta_{\Im}\right) \sin \left(\theta_{\Re}\right)|\theta|, \\
& \int_{0}^{2 \pi} \Im\left(\phi^{*}, \hat{H} \phi\right) \frac{d \theta_{\Re}}{d q} d q=\pi^{4} \exp \left(-\theta_{\Im}\right) \sin \left(\theta_{\Re}\right)|\theta|,
\end{aligned}
$$

such that

$$
\oint_{C}\left(\phi^{*}, \hat{H} \phi\right) d \theta=\oint_{C}\left(\hat{H} \phi^{*}, \phi\right) d \theta=0
$$

\section{HOLOMORPHICITY}

Cauchy's theorem states that if $t_{\sigma}(\theta)$ is an analytic function, and $t_{\sigma}^{\prime}(\theta)$ is continuous at each point within and on a closed contour $C$, then

$$
\oint_{C} t_{\sigma}(\theta) d \theta=0
$$

Theorem 10. Let $\theta=\theta_{\Re}+i \theta_{\Im}$ and $t_{\sigma}(\theta)=u_{\sigma}\left(\theta_{\Re}, \theta_{\Im}\right)+i v_{\sigma}\left(\theta_{\Re}, \theta_{\Im}\right)$ on some region $\mathbb{G}$ containing the point $\theta_{0}$. Then the eigenvalues $t_{\sigma}$ are holomorphic.

Proof. Integrating $\theta$ along $C$, Eq. 73 is then written

$$
\begin{aligned}
t_{\sigma} & =i \oint_{C}\left(\frac{|\sigma| \cos (\theta) \cos (2 \sigma \pi \cos (\theta))}{1-2|\sigma| \cos (\theta)}-\frac{|\sigma| \sin (\theta) \sin (2 \sigma \pi \cos (\theta))}{2|\sigma| \cos (\theta)-1}\right) d \theta \\
& -\oint_{C}\left(\frac{|\sigma| \cos (\theta) \sin (2 \sigma \pi \cos (\theta))}{2|\sigma| \cos (\theta)-1}+\frac{|\sigma| \cos (2 \sigma \pi \cos (\theta)) \sin (\theta)}{1-2|\sigma| \cos (\theta)}\right) d \theta \\
& +\frac{i}{2} \oint_{C} \frac{\cos (2 \sigma \pi \cos (\theta))}{2|\sigma| \cos (\theta)-1} d \theta-\frac{1}{2} \oint_{C} \frac{\sin (2 \sigma \pi \cos (\theta))}{1-2|\sigma| \cos (\theta)} d \theta .
\end{aligned}
$$

The path $C$ is then parametrized as follows:

$$
\theta(q)=|\theta| \cos (q)+i|\theta| \sin (q),
$$


with $0 \leq q \leq 2 \pi$, whilst $f(\theta)$ is given by Eq. (50), and the real and imaginary parts of Eq. 50 are given by Eqs. 52 and 53 , and

$$
|\theta|=\sqrt{\alpha^{2}+\beta^{2}}
$$

where

$$
\alpha=2|\sigma| \cos \left(\theta_{\Re}\right) \cosh \left(\theta_{\Im}\right)-1,
$$

and

$$
\beta=-2|\sigma| \sin \left(\theta_{\Re}\right) \sinh \left(\theta_{\Im}\right) .
$$

Here, it is useful to implement the following identities:

$$
\begin{aligned}
\cos \left(\theta_{\Re}+i \theta_{\Im}\right) & =\cos \left(\theta_{\Re}\right) \cosh \left(\theta_{\Im}\right)-i \sin \left(\theta_{\Re}\right) \sinh \left(\theta_{\Im}\right) \\
\cos \left(2 \sigma \pi \cos \left(\theta_{\Re}+i \theta_{\Im}\right)\right)= & \cos \left(2 \pi \sigma \cos \left(\theta_{\Re}\right) \cosh \left(\theta_{\Im}\right)\right) \cosh \left(2 \pi \sigma \sin \left(\theta_{\Re}\right) \sinh \left(\theta_{\Im}\right)\right) \\
+ & i \sinh \left(2 \pi \sigma \sin \left(\theta_{\Re}\right) \sinh \left(\theta_{\Im}\right)\right) \sin \left(2 \pi \sigma \cos \left(\theta_{\Re}\right) \cosh \left(\theta_{\Im}\right)\right),
\end{aligned}
$$

and

$$
\begin{aligned}
\sin \left(\theta_{\Re}+i \theta_{\Im}\right) & =\sin \left(\theta_{\Re}\right) \cosh \left(\theta_{\Im}\right)+i \cos \left(\theta_{\Re}\right) \sinh \left(\theta_{\Im}\right), \\
\sin \left(2 \sigma \pi \cos \left(\theta_{\Re}+i \theta_{\Im}\right)\right)= & \sin \left(2 \pi \sigma \cos \left(\theta_{\Re}\right) \cosh \left(\theta_{\Im}\right)\right) \cosh \left(2 \pi \sigma \sin \left(\theta_{\Re}\right) \sinh \left(\theta_{\Im}\right)\right) \\
& -i \sinh \left(2 \pi \sigma \sin \left(\theta_{\Re}\right) \sinh \left(\theta_{\Im}\right)\right) \cos \left(2 \pi \sigma \cos \left(\theta_{\Re}\right) \cosh \left(\theta_{\Im}\right)\right) .
\end{aligned}
$$

By performing the parametrization of the path $C$ as given by Eq. (111), the real and imaginary parts of $t_{\sigma}=u_{\sigma}+i v_{\sigma}$ are then

$$
\begin{aligned}
u_{\sigma} & =\frac{\pi}{|\theta|^{2}}\left(|\theta|^{2} \sin (\pi \alpha) \exp (\pi \beta)+2|\sigma| \beta \sin \left(\theta_{\Re}\right) \cosh \left(\theta_{\Im}\right) \exp (\pi \beta) \sin (\pi \alpha)\right. \\
& +2|\sigma| \alpha \sin \left(\theta_{\Re}\right) \cosh \left(\theta_{\Im}\right)+2|\sigma| \beta \cos \left(\theta_{\Re}\right) \sinh \left(\theta_{\Im}\right)-2|\sigma| \beta \cos \left(\theta_{\Re}\right) \sinh \left(\theta_{\Im}\right) \exp (\pi \beta) \cos (\pi \alpha) \\
& \left.-2|\sigma| \alpha \sin \left(\theta_{\Re}\right) \cosh \left(\theta_{\Im}\right) \exp (\pi \beta) \cos (\pi \alpha)-2|\sigma| \alpha \cos \left(\theta_{\Re}\right) \sinh \left(\theta_{\Im}\right) \exp (\pi \beta) \sin (\pi \alpha)\right),
\end{aligned}
$$

and

$$
\begin{aligned}
v_{\sigma} & =\frac{\pi}{|\theta|^{2}}\left(-|\theta|^{2}+|\theta|^{2} \exp (\pi \beta) \cos (\pi \alpha)+2|\sigma| \beta \sin \left(\theta_{\Re}\right) \cosh \left(\theta_{\Im}\right) \exp (\pi \beta) \cos (\pi \alpha)\right. \\
& +2|\sigma| \beta \cos \left(\theta_{\Re}\right) \sinh \left(\theta_{\Im}\right) \exp (\pi \beta) \sin (\pi \alpha)-2|\sigma| \alpha \sin \left(\theta_{\Re}\right) \cosh \left(\theta_{\Im}\right) \exp (\pi \beta) \sin (\pi \alpha) \\
& -2|\sigma| \alpha \cos \left(\theta_{\Re}\right) \sinh \left(\theta_{\Im}\right) \exp (\pi \beta) \cos (\pi \alpha)-4|\sigma| \sin \left(\theta_{\Re}\right) \cosh \left(\theta_{\Im}\right) \exp (\pi \beta) \sin (\pi \alpha) \\
& \left.-2|\sigma| \beta \sin \left(\theta_{\Re}\right) \cosh \left(\theta_{\Im}\right)+2|\sigma| \alpha \cos \left(\theta_{\Re}\right) \sinh \left(\theta_{\Im}\right)\right),
\end{aligned}
$$

where

$$
\begin{aligned}
u_{\sigma} & =\frac{2 \pi|\sigma| \sin \left(\theta_{\Re}\right) \sinh \left(\theta_{\Im}\right)}{1-4|\sigma| \cos \left(\theta_{\Re}\right) \cosh \left(\theta_{\Im}\right)+2 \sigma^{2}\left(\cos \left(2 \theta_{\Re}\right)+\cosh \left(2 \theta_{\Im}\right)\right)} \\
& +\frac{-4 \pi|\sigma|^{2} \cos \left(\theta_{\Re}\right) \cosh \left(\theta_{\Im}\right) \sin \left(\theta_{\Re}\right) \sinh \left(\theta_{\Im}\right) \exp \left(-2 \pi \sigma \sin \left(\theta_{\Re}\right) \sinh \left(\theta_{\Im}\right)\right) \cos \left(\pi-2 \pi \sigma \cos \left(\theta_{\Re}\right) \cosh \left(\theta_{\Im}\right)\right)}{1-4|\sigma| \cos \left(\theta_{\Re}\right) \cosh \left(\theta_{\Im}\right)+2 \sigma^{2}\left(\cos \left(2 \theta_{\Re}\right)+\cosh \left(2 \theta_{\Im}\right)\right)} \\
& +\frac{-4 \pi|\sigma|^{2} \cos ^{2}\left(\theta_{\Re}\right) \cosh ^{2}\left(\theta_{\Im}\right) \exp \left(-2 \pi \sigma \sin \left(\theta_{\Re}\right) \sinh \left(\theta_{\Im}\right)\right) \sin \left(\pi-2 \pi \sigma \cos \left(\theta_{\Re}\right) \cosh \left(\theta_{\Im}\right)\right)}{1-4|\sigma| \cos \left(\theta_{\Re}\right) \cosh \left(\theta_{\Im}\right)+2 \sigma^{2}\left(\cos \left(2 \theta_{\Re}\right)+\cosh \left(2 \theta_{\Im}\right)\right)}
\end{aligned}
$$




$$
\begin{aligned}
& +\frac{2 \pi|\sigma| \cos \left(\theta_{\Re}\right) \cosh \left(\theta_{\Im}\right) \exp \left(-2 \pi \sigma \sin \left(\theta_{\Re}\right) \sinh \left(\theta_{\Im}\right)\right) \sin \left(\pi-2 \pi \sigma \cos \left(\theta_{\Re}\right) \cosh \left(\theta_{\Im}\right)\right)}{1-4|\sigma| \cos \left(\theta_{\Re}\right) \cosh \left(\theta_{\Im}\right)+2 \sigma^{2}\left(\cos \left(2 \theta_{\Re}\right)+\cosh \left(2 \theta_{\Im}\right)\right)} \\
& +\frac{4 \pi|\sigma|^{2} \sin \left(\theta_{\Re}\right) \sinh \left(\theta_{\Im}\right) \cos \left(\theta_{\Re}\right) \cosh \left(\theta_{\Im}\right) \exp \left(-2 \pi \sigma \sin \left(\theta_{\Re}\right) \sinh \left(\theta_{\Im}\right)\right) \cos \left(\pi-2 \pi \sigma \cos \left(\theta_{\Re}\right) \cosh \left(\theta_{\Im}\right)\right)}{1-4|\sigma| \cos \left(\theta_{\Re}\right) \cosh \left(\theta_{\Im}\right)+2 \sigma^{2}\left(\cos \left(2 \theta_{\Re}\right)+\cosh \left(2 \theta_{\Im}\right)\right)} \\
& +\frac{-2 \pi|\sigma| \sin \left(\theta_{\Re}\right) \sinh \left(\theta_{\Im}\right) \exp \left(-2 \pi \sigma \sin \left(\theta_{\Re}\right) \sinh \left(\theta_{\Im}\right)\right) \cos \left(\pi-2 \pi \sigma \cos \left(\theta_{\Re}\right) \cosh \left(\theta_{\Im}\right)\right)}{1-4|\sigma| \cos \left(\theta_{\Re}\right) \cosh \left(\theta_{\Im}\right)+2 \sigma^{2}\left(\cos \left(2 \theta_{\Re}\right)+\cosh \left(2 \theta_{\Im}\right)\right)} \\
& +\frac{-4 \pi|\sigma|^{2} \sin ^{2}\left(\theta_{\Re}\right) \sinh ^{2}\left(\theta_{\Im}\right) \exp \left(-2 \pi \sigma \sin \left(\theta_{\Re}\right) \sinh \left(\theta_{\Im}\right)\right) \sin \left(\pi-2 \pi \sigma \cos \left(\theta_{\Re}\right) \cosh \left(\theta_{\Im}\right)\right)}{1-4|\sigma| \cos \left(\theta_{\Re}\right) \cosh \left(\theta_{\Im}\right)+2 \sigma^{2}\left(\cos \left(2 \theta_{\Re}\right)+\cosh \left(2 \theta_{\Im}\right)\right)} \\
& 1-4|\sigma| \cos \left(\theta_{\Re}\right) \cosh \left(\theta_{\Im}\right)+2 \sigma^{2}\left(\cos \left(2 \theta_{\Re}\right)+\cosh \left(2 \theta_{\Im}\right)\right) \\
& +\frac{-2 \pi|\sigma| \sin \left(\theta_{\Re}\right) \sinh \left(\theta_{\Im}\right) \exp \left(-2 \pi \sigma \sin \left(\theta_{\Re}\right) \sinh \left(\theta_{\Im}\right)\right) \cos \left(2 \pi-2 \pi \sigma \cos \left(\theta_{\Re}\right) \cosh \left(\theta_{\Im}\right)\right)}{1-4|\sigma| \cos \left(\theta_{\Re}\right) \cosh \left(\theta_{\Im}\right)+2 \sigma^{2}\left(\cos \left(2 \theta_{\Re}\right)+\cosh \left(2 \theta_{\Im}\right)\right)} \\
& +\frac{-2 \pi|\sigma| \cos \left(\theta_{\Re}\right) \cosh \left(\theta_{\Im}\right) \exp \left(-2 \pi \sigma \sin \left(\theta_{\Re}\right) \sinh \left(\theta_{\Im}\right)\right) \sin \left(2 \pi-2 \pi \sigma \cos \left(\theta_{\Re}\right) \cosh \left(\theta_{\Im}\right)\right)}{1-4|\sigma| \cos \left(\theta_{\Re}\right) \cosh \left(\theta_{\Im}\right)+2 \sigma^{2}\left(\cos \left(2 \theta_{\Re}\right)+\cosh \left(2 \theta_{\Im}\right)\right)} \\
& +\frac{\pi \exp \left(-2 \pi \sigma \sin \left(\theta_{\Re}\right) \sinh \left(\theta_{\Im}\right)\right) \sin \left(2 \pi-2 \pi \sigma \cos \left(\theta_{\Re}\right) \cosh \left(\theta_{\Im}\right)\right)}{1-4|\sigma| \cos \left(\theta_{\Re}\right) \cosh \left(\theta_{\Im}\right)+2 \sigma^{2}\left(\cos \left(2 \theta_{\Re}\right)+\cosh \left(2 \theta_{\Im}\right)\right)} \\
& +\frac{-4 \pi|\sigma|^{2} \sin \left(\theta_{\Re}\right) \cos \left(\theta_{\Re}\right) \cosh ^{2}\left(\theta_{\Im}\right) \exp \left(-2 \pi \sigma \sin \left(\theta_{\Re}\right) \sinh \left(\theta_{\Im}\right)\right) \cos \left(\pi-2 \pi \sigma \cos \left(\theta_{\Re}\right) \cosh \left(\theta_{\Im}\right)\right)}{1-4|\sigma| \cos \left(\theta_{\Re}\right) \cosh \left(\theta_{\Im}\right)+2 \sigma^{2}\left(\cos \left(2 \theta_{\Re}\right)+\cosh \left(2 \theta_{\Im}\right)\right)} \\
& +\frac{2 \pi|\sigma| \sin \left(\theta_{\Re}\right) \cosh \left(\theta_{\Im}\right) \exp \left(-2 \pi \sigma \sin \left(\theta_{\Re}\right) \sinh \left(\theta_{\Im}\right)\right) \cos \left(\pi-2 \pi \sigma \cos \left(\theta_{\Re}\right) \cosh \left(\theta_{\Im}\right)\right)}{1-4|\sigma| \cos \left(\theta_{\Re}\right) \cosh \left(\theta_{\Im}\right)+2 \sigma^{2}\left(\cos \left(2 \theta_{\Re}\right)+\cosh \left(2 \theta_{\Im}\right)\right)} \\
& +\frac{4 \pi|\sigma|^{2} \sin ^{2}\left(\theta_{\Re}\right) \cosh \left(\theta_{\Im}\right) \sinh \left(\theta_{\Im}\right) \exp \left(-2 \pi \sigma \sin \left(\theta_{\Re}\right) \sinh \left(\theta_{\Im}\right)\right) \sin \left(\pi-2 \pi \sigma \cos \left(\theta_{\Re}\right) \cosh \left(\theta_{\Im}\right)\right)}{1-4|\sigma| \cos \left(\theta_{\Re}\right) \cosh \left(\theta_{\Im}\right)+2 \sigma^{2}\left(\cos \left(2 \theta_{\Re}\right)+\cosh \left(2 \theta_{\Im}\right)\right)} \\
& +\frac{4 \pi|\sigma|^{2} \sin \left(\theta_{\Re}\right) \cos \left(\theta_{\Re}\right) \sinh ^{2}\left(\theta_{\Im}\right) \exp \left(-2 \pi \sigma \sin \left(\theta_{\Re}\right) \sinh \left(\theta_{\Im}\right)\right) \cos \left(\pi-2 \pi \sigma \cos \left(\theta_{\Re}\right) \cosh \left(\theta_{\Im}\right)\right)}{1-4|\sigma| \cos \left(\theta_{\Re}\right) \cosh \left(\theta_{\Im}\right)+2 \sigma^{2}\left(\cos \left(2 \theta_{\Re}\right)+\cosh \left(2 \theta_{\Im}\right)\right)} \\
& +\frac{4 \pi|\sigma|^{2} \cos ^{2}\left(\theta_{\Re}\right) \sinh \left(\theta_{\Im}\right) \cosh \left(\theta_{\Im}\right) \exp \left(-2 \pi \sigma \sin \left(\theta_{\Re}\right) \sinh \left(\theta_{\Im}\right)\right) \sin \left(\pi-2 \pi \sigma \cos \left(\theta_{\Re}\right) \cosh \left(\theta_{\Im}\right)\right)}{1-4|\sigma| \cos \left(\theta_{\Re}\right) \cosh \left(\theta_{\Im}\right)+2 \sigma^{2}\left(\cos \left(2 \theta_{\Re}\right)+\cosh \left(2 \theta_{\Im}\right)\right)} \\
& +\frac{-2 \pi|\sigma| \cos \left(\theta_{\Re}\right) \sinh \left(\theta_{\Im}\right) \exp \left(-2 \pi \sigma \sin \left(\theta_{\Re}\right) \sinh \left(\theta_{\Im}\right)\right) \sin \left(\pi-2 \pi \sigma \cos \left(\theta_{\Re}\right) \cosh \left(\theta_{\Im}\right)\right)}{1-4|\sigma| \cos \left(\theta_{\Re}\right) \cosh \left(\theta_{\Im}\right)+2 \sigma^{2}\left(\cos \left(2 \theta_{\Re}\right)+\cosh \left(2 \theta_{\Im}\right)\right)} \\
& +\frac{-2 \pi|\sigma| \sin \left(\theta_{\Re}\right) \sinh \left(\theta_{\Im}\right)+4 \pi|\sigma|^{2} \sin \left(\theta_{\Re}\right) \cosh ^{2}\left(\theta_{\Im}\right) \cos \left(\theta_{\Re}\right)}{1-4|\sigma| \cos \left(\theta_{\Re}\right) \cosh \left(\theta_{\Im}\right)+2 \sigma^{2}\left(\cos \left(2 \theta_{\Re}\right)+\cosh \left(2 \theta_{\Im}\right)\right)} \\
& +\frac{-2 \pi|\sigma| \sin \left(\theta_{\Re}\right) \cosh \left(\theta_{\Im}\right)-4 \pi|\sigma|^{2} \cos \left(\theta_{\Re}\right) \sin \left(\theta_{\Re}\right) \sinh ^{2}\left(\theta_{\Im}\right)}{1-4|\sigma| \cos \left(\theta_{\Re}\right) \cosh \left(\theta_{\Im}\right)+2 \sigma^{2}\left(\cos \left(2 \theta_{\Re}\right)+\cosh \left(2 \theta_{\Im}\right)\right)},
\end{aligned}
$$


and

$$
\begin{aligned}
& v_{\sigma}=\frac{-4 \pi|\sigma|^{2} \cos ^{2}\left(\theta_{\Re}\right) \cosh ^{2}\left(\theta_{\Im}\right)-4 \pi|\sigma|^{2} \sin ^{2}\left(\theta_{\Re}\right) \sinh ^{2}\left(\theta_{\Im}\right)}{1-4|\sigma| \cos \left(\theta_{\Re}\right) \cosh \left(\theta_{\Im}\right)+2 \sigma^{2}\left(\cos \left(2 \theta_{\Re}\right)+\cosh \left(2 \theta_{\Im}\right)\right)} \\
& +\frac{4 \pi|\sigma| \cos \left(\theta_{\Re}\right) \cosh \left(\theta_{\Im}\right)-\pi}{1-4|\sigma| \cos \left(\theta_{\Re}\right) \cosh \left(\theta_{\Im}\right)+2 \sigma^{2}\left(\cos \left(2 \theta_{\Re}\right)+\cosh \left(2 \theta_{\Im}\right)\right)} \\
& +\frac{4 \pi|\sigma|^{2} \cos ^{2}\left(\theta_{\Re}\right) \cosh ^{2}\left(\theta_{\Im}\right) \exp \left(-2 \pi \sigma \sin \left(\theta_{\Re}\right) \sinh \left(\theta_{\Im}\right)\right) \cos \left(\pi-2 \pi \sigma \cos \left(\theta_{\Re}\right) \cosh \left(\theta_{\Im}\right)\right)}{1-4|\sigma| \cos \left(\theta_{\Re}\right) \cosh \left(\theta_{\Im}\right)+2 \sigma^{2}\left(\cos \left(2 \theta_{\Re}\right)+\cosh \left(2 \theta_{\Im}\right)\right)} \\
& +\frac{-2 \pi|\sigma| \cos \left(\theta_{\Re}\right) \cosh \left(\theta_{\Im}\right) \exp \left(-2 \pi \sigma \sin \left(\theta_{\Re}\right) \sinh \left(\theta_{\Im}\right)\right) \cos \left(\pi-2 \pi \sigma \cos \left(\theta_{\Re}\right) \cosh \left(\theta_{\Im}\right)\right)}{1-4|\sigma| \cos \left(\theta_{\Re}\right) \cosh \left(\theta_{\Im}\right)+2 \sigma^{2}\left(\cos \left(2 \theta_{\Re}\right)+\cosh \left(2 \theta_{\Im}\right)\right)} \\
& +\frac{4 \pi|\sigma|^{2} \sin ^{2}\left(\theta_{\Re}\right) \sinh ^{2}\left(\theta_{\Im}\right) \exp \left(-2 \pi \sigma \sin \left(\theta_{\Re}\right) \sinh \left(\theta_{\Im}\right)\right) \cos \left(\pi-2 \pi \sigma \cos \left(\theta_{\Re}\right) \cosh \left(\theta_{\Im}\right)\right)}{1-4|\sigma| \cos \left(\theta_{\Re}\right) \cosh \left(\theta_{\Im}\right)+2 \sigma^{2}\left(\cos \left(2 \theta_{\Re}\right)+\cosh \left(2 \theta_{\Im}\right)\right)} \\
& +\frac{-2 \pi|\sigma| \sin \left(\theta_{\Re}\right) \sinh \left(\theta_{\Im}\right) \exp \left(-2 \pi \sigma \sin \left(\theta_{\Re}\right) \sinh \left(\theta_{\Im}\right)\right) \sin \left(\pi-2 \pi \sigma \cos \left(\theta_{\Re}\right) \cosh \left(\theta_{\Im}\right)\right)}{1-4|\sigma| \cos \left(\theta_{\Re}\right) \cosh \left(\theta_{\Im}\right)+2 \sigma^{2}\left(\cos \left(2 \theta_{\Re}\right)+\cosh \left(2 \theta_{\Im}\right)\right)} \\
& +\frac{2 \pi|\sigma| \cos \left(\theta_{\Re}\right) \cosh \left(\theta_{\Im}\right) \exp \left(-2 \pi \sigma \sin \left(\theta_{\Re}\right) \sinh \left(\theta_{\Im}\right)\right) \cos \left(2 \pi-2 \pi \sigma \cos \left(\theta_{\Re}\right) \cosh \left(\theta_{\Im}\right)\right)}{1-4|\sigma| \cos \left(\theta_{\Re}\right) \cosh \left(\theta_{\Im}\right)+2 \sigma^{2}\left(\cos \left(2 \theta_{\Re}\right)+\cosh \left(2 \theta_{\Im}\right)\right)} \\
& +\frac{-\pi \exp \left(-2 \pi \sigma \sin \left(\theta_{\Re}\right) \sinh \left(\theta_{\Im}\right)\right) \cos \left(2 \pi-2 \pi \sigma \cos \left(\theta_{\Re}\right) \cosh \left(\theta_{\Im}\right)\right)}{1-4|\sigma| \cos \left(\theta_{\Re}\right) \cosh \left(\theta_{\Im}\right)+2 \sigma^{2}\left(\cos \left(2 \theta_{\Re}\right)+\cosh \left(2 \theta_{\Im}\right)\right)} \\
& +\frac{-2 \pi|\sigma| \sin \left(\theta_{\Re}\right) \sinh \left(\theta_{\Im}\right) \exp \left(-2 \pi \sigma \sin \left(\theta_{\Re}\right) \sinh \left(\theta_{\Im}\right)\right) \sin \left(2 \pi-2 \pi \sigma \cos \left(\theta_{\Re}\right) \cosh \left(\theta_{\Im}\right)\right)}{1-4|\sigma| \cos \left(\theta_{\Re}\right) \cosh \left(\theta_{\Im}\right)+2 \sigma^{2}\left(\cos \left(2 \theta_{\Re}\right)+\cosh \left(2 \theta_{\Im}\right)\right)} \\
& +\frac{-4 \pi|\sigma|^{2} \sin ^{2}\left(\theta_{\Re}\right) \cosh \left(\theta_{\Im}\right) \sinh \left(\theta_{\Im}\right) \exp \left(-2 \pi \sigma \sin \left(\theta_{\Re}\right) \sinh \left(\theta_{\Im}\right)\right) \cos \left(\pi-2 \pi \sigma \cos \left(\theta_{\Re}\right) \cosh \left(\theta_{\Im}\right)\right)}{1-4|\sigma| \cos \left(\theta_{\Re}\right) \cosh \left(\theta_{\Im}\right)+2 \sigma^{2}\left(\cos \left(2 \theta_{\Re}\right)+\cosh \left(2 \theta_{\Im}\right)\right)} \\
& +\frac{-4 \pi|\sigma|^{2} \sin \left(\theta_{\Re}\right) \cos \left(\theta_{\Re}\right) \cosh ^{2}\left(\theta_{\Im}\right) \exp \left(-2 \pi \sigma \sin \left(\theta_{\Re}\right) \sinh \left(\theta_{\Im}\right)\right) \sin \left(\pi-2 \pi \sigma \cos \left(\theta_{\Re}\right) \cosh \left(\theta_{\Im}\right)\right)}{1-4|\sigma| \cos \left(\theta_{\Re}\right) \cosh \left(\theta_{\Im}\right)+2 \sigma^{2}\left(\cos \left(2 \theta_{\Re}\right)+\cosh \left(2 \theta_{\Im}\right)\right)} \\
& +\frac{2 \pi|\sigma| \sin \left(\theta_{\Re}\right) \cosh \left(\theta_{\Im}\right) \exp \left(-2 \pi \sigma \sin \left(\theta_{\Re}\right) \sinh \left(\theta_{\Im}\right)\right) \sin \left(\pi-2 \pi \sigma \cos \left(\theta_{\Re}\right) \cosh \left(\theta_{\Im}\right)\right)}{1-4|\sigma| \cos \left(\theta_{\Re}\right) \cosh \left(\theta_{\Im}\right)+2 \sigma^{2}\left(\cos \left(2 \theta_{\Re}\right)+\cosh \left(2 \theta_{\Im}\right)\right)} \\
& +\frac{-4 \pi|\sigma|^{2} \cos ^{2}\left(\theta_{\Re}\right) \sinh \left(\theta_{\Im}\right) \exp \left(-2 \pi \sigma \sin \left(\theta_{\Re}\right) \sinh \left(\theta_{\Im}\right)\right) \cos \left(\pi-2 \pi \sigma \cos \left(\theta_{\Re}\right) \cosh \left(\theta_{\Im}\right)\right)}{1-4|\sigma| \cos \left(\theta_{\Re}\right) \cosh \left(\theta_{\Im}\right)+2 \sigma^{2}\left(\cos \left(2 \theta_{\Re}\right)+\cosh \left(2 \theta_{\Im}\right)\right)} \\
& +\frac{2 \pi|\sigma| \cos \left(\theta_{\Re}\right) \sinh \left(\theta_{\Im}\right) \exp \left(-2 \pi \sigma \sin \left(\theta_{\Re}\right) \sinh \left(\theta_{\Im}\right)\right) \cos \left(\pi-2 \pi \sigma \cos \left(\theta_{\Re}\right) \cosh \left(\theta_{\Im}\right)\right)}{1-4|\sigma| \cos \left(\theta_{\Re}\right) \cosh \left(\theta_{\Im}\right)+2 \sigma^{2}\left(\cos \left(2 \theta_{\Re}\right)+\cosh \left(2 \theta_{\Im}\right)\right)} \\
& +\frac{4 \pi|\sigma|^{2} \cos \left(\theta_{\Re}\right) \sin \left(\theta_{\Re}\right) \sinh ^{2}\left(\theta_{\Im}\right) \exp \left(-2 \pi \sigma \sin \left(\theta_{\Re}\right) \sinh \left(\theta_{\Im}\right)\right) \sin \left(\pi-2 \pi \sigma \cos \left(\theta_{\Re}\right) \cosh \left(\theta_{\Im}\right)\right)}{1-4|\sigma| \cos \left(\theta_{\Re}\right) \cosh \left(\theta_{\Im}\right)+2 \sigma^{2}\left(\cos \left(2 \theta_{\Re}\right)+\cosh \left(2 \theta_{\Im}\right)\right)}
\end{aligned}
$$




$$
+\frac{4 \pi|\sigma|^{2} \sin ^{2}\left(\theta_{\Re}\right) \cosh \left(\theta_{\Im}\right) \sinh \left(\theta_{\Im}\right)+4 \pi|\sigma|^{2} \cos ^{2}\left(\theta_{\Re}\right) \sinh \left(\theta_{\Im}\right) \cosh \left(\theta_{\Im}\right)-2 \pi|\sigma| \cos \left(\theta_{\Re}\right) \sinh \left(\theta_{\Im}\right)}{1-4|\sigma| \cos \left(\theta_{\Re}\right) \cosh \left(\theta_{\Im}\right)+2 \sigma^{2}\left(\cos \left(2 \theta_{\Re}\right)+\cosh \left(2 \theta_{\Im}\right)\right)} .
$$

Hence, from the relation

$$
\begin{aligned}
\oint_{C} t_{\sigma}(\theta) d \theta & =\oint_{C}\left(u_{\sigma}+i v_{\sigma}\right)\left(d \theta_{\Re}+i d \theta_{\Im}\right) \\
& =\oint_{C} u_{\sigma} d \theta_{\Re}-\oint_{C} v_{\sigma} d \theta_{\Im}+i \oint_{C} u_{\sigma} d \theta_{\Im}+i \oint_{C} v_{\sigma} d \theta_{\Re} \\
& =\int_{0}^{2 \pi} u_{\sigma} \frac{d \theta_{\Re}}{d q} d q-\int_{0}^{2 \pi} v_{\sigma} \frac{d \theta_{\Im}}{d q} d q+i \int_{0}^{2 \pi} u_{\sigma} \frac{d \theta_{\Im}}{d q} d q+i \int_{0}^{2 \pi} v_{\sigma} \frac{d \theta_{\Re}}{d q} d q
\end{aligned}
$$

at $|\theta|=1$, we obtain

$$
\begin{aligned}
\int_{0}^{2 \pi} u_{\sigma} \frac{d \theta_{\Re}}{d q} d q & =\pi^{4}|\sigma| \cos \left(\theta_{\Re}\right) \sinh \left(\theta_{\Im}\right), \\
\int_{0}^{2 \pi} v_{\sigma} \frac{d \theta_{\Im}}{d q} d q & =\pi^{3}|\sigma|\left(\pi \cos \left(\theta_{\Re}\right) \sinh \left(\theta_{\Im}\right)-4 \sin \left(\theta_{\Re}\right) \cosh \left(\theta_{\Im}\right)\right), \\
\int_{0}^{2 \pi} u_{\sigma} \frac{d \theta_{\Im}}{d q} d q & =\frac{1}{2} \pi^{3}\left((4+\pi) \sin \left(\theta_{\Re}\right)|\sigma| \cosh \left(\theta_{\Im}\right)+2\right), \\
\int_{0}^{2 \pi} v_{\sigma} \frac{d \theta_{\Re}}{d q} d q & =-\frac{1}{2} \pi^{2}\left(4+\pi^{2}\right) \sin \left(\theta_{\Re}\right)|\sigma| \cosh \left(\theta_{\Im}\right)+\frac{1}{2} \pi^{4} \sin \left(\theta_{\Re}\right)|\sigma| \cosh \left(\theta_{\Im}\right) \\
& +2 \pi^{2} \sin \left(\theta_{\Re}\right)|\sigma| \cosh \left(\theta_{\Im}\right)-\pi^{3}
\end{aligned}
$$

such that

$$
\begin{aligned}
\oint_{C} t_{\sigma}(\theta) d \theta & =\frac{i \pi^{3}}{2} \int_{\sin ^{-1}}^{\sin ^{-1}\left(-\frac{\pi}{2|s|}\right)+2 \pi \sigma} \int_{\cos ^{-1}\left(\frac{1}{2|s|}\right)}^{\cos ^{-1}\left(\frac{1}{2|s|}\right)+2 \pi \sigma}(\pi+(4-8 i)) \sin \left(\theta_{\Re}\right)|\sigma| \cosh \left(\theta_{\Im}\right) d \theta_{\Re} d \theta_{\Im} \\
& =2(8+4 i+i \pi) \pi^{3}|\sigma| \sin (\pi \sigma) \sinh (\pi \sigma) \cosh \left(\pi \sigma-\sin ^{-1}\left(\frac{\pi}{2|s|}\right)\right) \\
& \cdot \cos \left(i \log \left(\frac{\sqrt{4 s s^{*}-1}+i}{|s|}\right)+\pi \sigma-i \log (2)\right) \\
& =0 \forall \sigma .
\end{aligned}
$$

Here, it should be pointed out, that in accordance with Eq. (38), when $|\sigma|=1 / 2$, and since $\theta_{\Im}=0$, Eq. (121) then reduces to

$$
u_{\sigma}(\theta)=-\pi \sin (\pi \cos (\theta))-\pi(\cos (\pi \cos (\theta))+1) \cot \left(\frac{\theta}{2}\right)
$$

and Eq. 122 then reduces to

$$
v_{\sigma}(\theta)=-\pi\left(\cos (\pi \cos (\theta))-\cot \left(\frac{\theta}{2}\right) \sin (\pi \cos (\theta))+1\right),
$$

such that $t_{\sigma}=u_{\sigma}+i v_{\sigma}$ in agreement with Eq. 74.

Theorem 11. Imaginary solutions of $\hbar \partial_{s}\left|\phi_{s}(x)\right\rangle=-2 \sqrt{x} \partial_{x} \sqrt{x}\left|\phi_{s}(x)\right\rangle$ at $x=1$ map to the nontrivial zeros of $\zeta(s)=\left(1-2^{1-s}\right)^{-1} \sum_{n=1}^{\infty}(-1)^{n-1} n^{-s}$.

Proof. At $x=1$, the normalization constraint Eq. (54) is satisfied, $\sigma=\frac{1}{2}-i t$, and Eq. (4) can be written 


$$
\begin{aligned}
\Psi_{s}(x=1) & =-\zeta(\sigma, 2) \\
& =-\Gamma(1-s) \frac{1}{2 \pi i} \oint_{C} \frac{z^{s-1} e^{2 z}}{1-e^{z}} d z \\
& =1-\zeta\left(\sigma=\frac{1}{2}-i t\right) .
\end{aligned}
$$

where the contour $C$ is about $\mathbb{R}^{-}$. From the analytic continuation relations of Eq. (1)

$$
\begin{aligned}
\frac{1}{1-2^{1-s}} \sum_{n=1}^{\infty} \frac{(-1)^{n-1}}{n^{s}} & =\frac{1}{1-2^{1-s}} \sum_{n=1}^{\infty} \frac{(-1)^{n-1} \exp (-i \cdot t \ln (n))}{n^{\sigma}} \\
& =\frac{1}{1-2^{1-s}}\left[\sum_{n=1}^{\infty} \frac{(-1)^{n-1} \cos (t \cdot \ln (n))}{n^{\sigma}}-i \sum_{n=1}^{\infty} \frac{(-1)^{n-1} \sin (t \cdot \ln (n))}{n^{\sigma}}\right] \\
1-\left(\frac{1}{1-2^{1-s}} \sum_{n=1}^{\infty} \frac{(-1)^{n-1}}{n^{s}}\right)^{*} & =1-\frac{1}{1-2^{1-s^{*}}} \sum_{n=1}^{\infty} \frac{(-1)^{n-1} \exp (i \cdot t \ln (n))}{n^{\sigma}} \\
& =1-\frac{1}{1-2^{1-s^{*}}}\left[\sum_{n=1}^{\infty} \frac{(-1)^{n-1} \cos (t \cdot \ln (n))}{n^{\sigma}}\right. \\
& \left.+i \sum_{n=1}^{\infty} \frac{(-1)^{n-1} \sin (t \cdot \ln (n))}{n^{\sigma}}\right]
\end{aligned}
$$

Owing to $t_{\sigma}=u_{\sigma}+i v_{\sigma}$ and Eqs. 115) and (117), it can be seen that

$$
\begin{aligned}
\frac{1}{1-2^{1-s}} \sum_{n=1}^{\infty} \frac{(-1)^{n-1}}{n^{s}} & =\sum_{n=1}^{\infty} \frac{(-1)^{n-1}}{n^{\sigma}} \cdot \frac{-2^{-\sigma+v_{\sigma}+1} \cos \left(u_{\sigma} \log (2)\right) \cos \left(u_{\sigma} \ln (n)\right) \exp \left(v_{\sigma} \ln (n)\right)}{2^{-2 \sigma+2 v_{\sigma}+2} \sin ^{2}\left(u_{\sigma} \log (2)\right)+\left[1-2^{-\sigma+v_{\sigma}+1} \cos \left(u_{\sigma} \log (2)\right)\right]^{2}} \\
& +\sum_{n=1}^{\infty} \frac{(-1)^{n-1}}{n^{\sigma}} \cdot \frac{\cos \left(u_{\sigma} \ln (n)\right) \exp \left(v_{\sigma} \ln (n)\right)}{2^{-2 \sigma+2 v_{\sigma}+2} \sin ^{2}\left(u_{\sigma} \log (2)\right)+\left[1-2^{-\sigma+v_{\sigma}+1} \cos \left(u_{\sigma} \log (2)\right)\right]^{2}} \\
& +\sum_{n=1}^{\infty} \frac{(-1)^{n-1}}{n^{\sigma}} \cdot \frac{-2^{-\sigma+v_{\sigma}+1} \sin \left(u_{\sigma} \log (2)\right) \sin \left(u_{\sigma} \ln (n)\right) \exp \left(v_{\sigma} \ln (n)\right)}{2^{-2 \sigma+2 v_{\sigma}+2} \sin ^{2}\left(u_{\sigma} \log (2)\right)+\left[1-2^{-\sigma+v_{\sigma}+1} \cos \left(u_{\sigma} \log (2)\right)\right]^{2}} \\
& +i \sum_{n=1}^{\infty} \frac{(-1)^{n-1}}{n^{\sigma}} \cdot \frac{-2^{-\sigma+v_{\sigma}+1} \sin \left(u_{\sigma} \log (2)\right) \cos \left(u_{\sigma} \ln (n)\right) \exp \left(v_{\sigma} \ln (n)\right)}{2^{-2 \sigma+2 v_{\sigma}+2} \sin ^{2}\left(u_{\sigma} \log (2)\right)+\left[1-2^{-\sigma+v_{\sigma}+1} \cos \left(u_{\sigma} \log (2)\right)\right]^{2}} \\
& +i \sum_{n=1}^{\infty} \frac{(-1)^{n-1}}{n^{\sigma}} \cdot \frac{2^{-\sigma+v_{\sigma}+1} \cos \left(u_{\sigma} \log (2)\right) \sin \left(u_{\sigma} \ln (n)\right) \exp \left(v_{\sigma} \ln (n)\right)}{2^{-2 \sigma+2 v_{\sigma}+2} \sin ^{2}\left(u_{\sigma} \log (2)\right)+\left[1-2^{-\sigma+v_{\sigma}+1} \cos \left(u_{\sigma} \log (2)\right)\right]^{2}} \\
& +i \sum_{n=1}^{\infty} \frac{(-1)^{n-1}}{n^{\sigma}} \cdot \frac{-\sin \left(u_{\sigma} \ln (n)\right) \exp \left(v_{\sigma} \ln (n)\right)}{2^{-2 \sigma+2 v_{\sigma}+2} \sin ^{2}\left(u_{\sigma} \log (2)\right)+\left[1-2^{-\sigma+v_{\sigma}+1} \cos \left(u_{\sigma} \log (2)\right)\right]^{2}} .
\end{aligned}
$$

and

$$
\begin{aligned}
1-\left(\frac{1}{1-2^{1-s}} \sum_{n=1}^{\infty} \frac{(-1)^{n-1}}{n^{s}}\right)^{*} & =1 \\
& +\sum_{n=1}^{\infty} \frac{(-1)^{n-1}}{n^{\sigma}} \cdot \frac{2^{-\sigma-v_{\sigma}+1} \cos \left(u_{\sigma} \log (2)\right) \cos \left(u_{\sigma} \ln (n)\right) \exp \left(-v_{\sigma} \ln (n)\right)}{2^{-2 \sigma-2 v_{\sigma}+2} \sin ^{2}\left(u_{\sigma} \log (2)\right)+\left[1-2^{-\sigma-v_{\sigma}+1} \cos \left(u_{\sigma} \log (2)\right)\right]^{2}}
\end{aligned}
$$




$$
\begin{aligned}
& +\sum_{n=1}^{\infty} \frac{(-1)^{n-1}}{n^{\sigma}} \cdot \frac{-\cos \left(u_{\sigma} \ln (n)\right) \exp \left(-v_{\sigma} \ln (n)\right)}{2^{-2 \sigma-2 v_{\sigma}+2} \sin ^{2}\left(u_{\sigma} \log (2)\right)+\left[1-2^{-\sigma-v_{\sigma}+1} \cos \left(u_{\sigma} \log (2)\right)\right]^{2}} \\
& +\sum_{n=1}^{\infty} \frac{(-1)^{n-1}}{n^{\sigma}} \cdot \frac{-2^{-\sigma-v_{\sigma}+1} \sin \left(u_{\sigma} \log (2)\right) \sin \left(u_{\sigma} \ln (n)\right) \exp \left(-v_{\sigma} \ln (n)\right)}{2^{-2 \sigma-2 v_{\sigma}+2} \sin ^{2}\left(u_{\sigma} \log (2)\right)+\left[1-2^{-\sigma-v_{\sigma}+1} \cos \left(u_{\sigma} \log (2)\right)\right]^{2}} \\
& +i \sum_{n=1}^{\infty} \frac{(-1)^{n-1}}{n^{\sigma}} \cdot \frac{-2^{-\sigma-v_{\sigma}+1} \sin \left(u_{\sigma} \log (2)\right) \cos \left(u_{\sigma} \ln (n)\right) \exp \left(-v_{\sigma} \ln (n)\right)}{2^{-2 \sigma-2 v_{\sigma}+2} \sin ^{2}\left(u_{\sigma} \log (2)\right)+\left[1-2^{-\sigma-v_{\sigma}+1} \cos \left(u_{\sigma} \log (2)\right)\right]^{2}} \\
& +i \sum_{n=1}^{\infty} \frac{(-1)^{n-1}}{n^{\sigma}} \cdot \frac{2^{-\sigma-v_{\sigma}+1} \cos ^{2}\left(u_{\sigma} \log (2)\right) \sin \left(u_{\sigma} \ln (n)\right) \exp \left(-v_{\sigma} \ln (n)\right)}{2^{-2 \sigma-2 v_{\sigma}+2} \sin ^{2}\left(u_{\sigma} \log (2)\right)+\left[1-2^{-\sigma-v_{\sigma}+1} \cos \left(u_{\sigma} \log (2)\right)\right]^{2}} \\
& +i \sum_{n=1}^{\infty} \frac{(-1)^{n-1}}{n^{\sigma}} \cdot \frac{-\sin \left(u_{\sigma} \ln (n)\right) \exp \left(-v_{\sigma} \ln (n)\right)}{2^{-2 \sigma-2 v_{\sigma}+2} \sin ^{2}\left(u_{\sigma} \log (2)\right)+\left[1-2^{-\sigma-v_{\sigma}+1} \cos \left(u_{\sigma} \log (2)\right)\right]^{2}}
\end{aligned}
$$

such that

$$
\Im\left[\frac{1}{1-2^{1-s}} \sum_{n=1}^{\infty} \frac{(-1)^{n-1}}{n^{s}}\right]=\Im\left[1-\left(\frac{1}{1-2^{1-s}} \sum_{n=1}^{\infty} \frac{(-1)^{n-1}}{n^{s}}\right)^{*}\right] \Longleftrightarrow v_{\sigma}=0 .
$$

Finally, the real part of every nontrivial zero of Eq. (1) can be obtained from setting the imaginary part of Eq. (134) and $v_{\sigma}$ to zero, i.e.,

$$
\begin{aligned}
\Im\left(\frac{1}{1-2^{1-s}} \sum_{n=1}^{\infty} \frac{(-1)^{n-1}}{n^{s}}\right) & =i \sum_{n=1}^{\infty} \frac{(-1)^{n-1}}{n^{\sigma}} \cdot \frac{-2^{-\sigma+1} \sin \left(u_{\sigma} \log (2)\right) \cos \left(u_{\sigma} \ln (n)\right)}{2^{-2 \sigma+2} \sin ^{2}\left(u_{\sigma} \log (2)\right)+\left[1-2^{-\sigma+1} \cos \left(u_{\sigma} \log (2)\right)\right]^{2}} \\
& +i \sum_{n=1}^{\infty} \frac{(-1)^{n-1}}{n^{\sigma}} \cdot \frac{2^{-\sigma+1} \cos \left(u_{\sigma} \log (2)\right) \sin \left(u_{\sigma} \ln (n)\right)}{2^{-2 \sigma+2} \sin ^{2}\left(u_{\sigma} \log (2)\right)+\left[1-2^{-\sigma+1} \cos \left(u_{\sigma} \log (2)\right)\right]^{2}} \\
& +i \sum_{n=1}^{\infty} \frac{(-1)^{n-1}}{n^{\sigma}} \cdot \frac{-\sin \left(u_{\sigma} \ln (n)\right)}{2^{-2 \sigma+2} \sin ^{2}\left(u_{\sigma} \log (2)\right)+\left[1-2^{-\sigma+1} \cos \left(u_{\sigma} \log (2)\right)\right]^{2}} \\
& =0 .
\end{aligned}
$$

Owing to Eq. (38), at $\sigma=1 / 2$ we obtain

$$
i \sum_{n=1}^{\infty} \frac{(-1)^{n-1}}{\sqrt{n}} \cdot \frac{\sin \left(u_{\sigma} \ln (n)\right)-\sqrt{2} \sin \left(u_{\sigma} \log \left(\frac{n}{2}\right)\right)}{2 \sqrt{2} \cos \left(u_{\sigma} \log (2)\right)-3}=0 .
$$

Since $v_{\sigma}=0$, then according to Eq. $130, \theta=(2 n-1) \pi$. Furthermore, from Eq. (129) it can be seen that at $\theta=(2 n-1) \pi, u_{\sigma}=0$, and the LHS of Eq. 138 is then $0 \forall n$.

Remark. The Riemann Hypothesis states that the real part of all of the nontrivial zeros of the Riemann zeta function are located at $\sigma=1 / 2$ [8].

Remark. Solutions to Eq. (3) are symmetric about the origin, i.e., $x \in[1, \infty)$, [-1, $-\infty)$, and subject to the singularity at $\phi_{s}(x=0)=0$ [25]. 


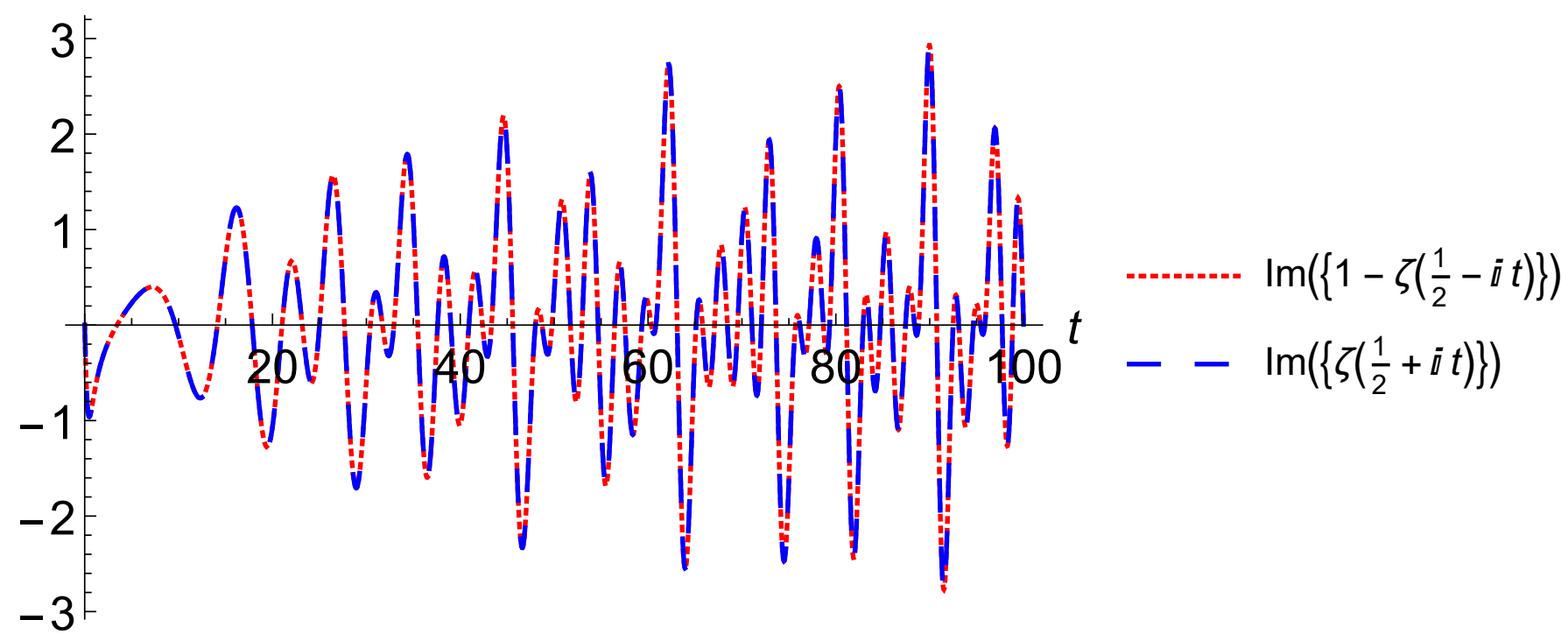

Figure 1: Plot of the imaginary components of Eq. (1). Results are compared with Eq. 138 (color online).

The expectation value of $\hat{H}$ can be expressed via the density operator, viz.,

$$
\begin{aligned}
& \langle\hat{H}\rangle(s)=\sum_{m} \sum_{n} \hat{b}_{m}^{\dagger}(s) \hat{b}_{n}(s) \frac{1-2 e^{i \theta}|n|}{e^{-i \theta}|m|+e^{i \theta}|n|-1}|m, n\rangle \\
& +\sum_{m} \sum_{n} \hat{b}_{m}^{\dagger}(s) \hat{b}_{n}(s) \frac{e^{i \theta}\left(1-2 e^{i \theta}|n|\right)(-1)^{-e^{-i \theta}\left(|m|+e^{2 i \theta}|n|\right)}}{|m|+e^{2 i \theta}|n|-e^{i \theta}}|m, n\rangle \\
& =\sum_{m} \sum_{n} \hat{\rho}_{m n}(s) \frac{1-2 e^{i \theta}|n|}{e^{-i \theta}|m|+e^{i \theta}|n|-1}|m, n\rangle \\
& +\sum_{m} \sum_{n} \hat{\rho}_{m n}(s) \frac{e^{i \theta}\left(1-2 e^{i \theta}|n|\right)(-1)^{-e^{-i \theta}\left(|m|+e^{2 i \theta}|n|\right)}}{|m|+e^{2 i \theta}|n|-e^{i \theta}}|m, n\rangle \\
& =\operatorname{Tr}\left[\hat{\rho}(s)\left(\frac{1-2 e^{i \theta}|n|}{e^{-i \theta}|n|+e^{i \theta}|n|-1}|n\rangle+\frac{e^{i \theta}(-1)^{-e^{-i \theta}\left(|n|+e^{2 i \theta}|n|\right)}\left(1-2 e^{i \theta}|n|\right)}{e^{2 i \theta}|n|+|n|-e^{i \theta}}|n\rangle\right)\right] \\
& =i \pi \sum_{n=1}^{\infty}\left(\frac{1-2 e^{i \theta}|n|}{e^{-i \theta}|n|+e^{i \theta}|n|-1}|n\rangle+\frac{e^{i \theta}(-1)^{-e^{-i \theta}\left(|n|+e^{2 i \theta}|n|\right)}\left(1-2 e^{i \theta}|n|\right)}{e^{2 i \theta}|n|+|n|-e^{i \theta}}|n\rangle\right) \text {. }
\end{aligned}
$$

\section{SIMILARITY SOLUTIONS}

Since Eq. (32), the Riemann zeta Schrödinger equation (RZSE) possesses symmetry about the origin $x=0$, we then seek a similarity solution [34] of the form:

$$
\phi_{s}(x)=x^{\alpha} f(\eta)
$$

where $\eta=s / x^{\beta}$, and the RZSE becomes an ordinary differential equation (ODE) for $f$. As such, we consider Eq. (32), and introduce the transformation $\xi=\epsilon^{a} x$, and $\tau=\epsilon^{b} s$, so that

$$
w(\xi, \tau)=\epsilon^{c} \phi\left(\epsilon^{-a} \xi, \epsilon^{-b} \tau\right)
$$

where $\epsilon \in \mathbb{R}$, and $\tau \in \mathbb{C}$. 
From performing this change of variable we obtain

$$
\begin{aligned}
\frac{\partial}{\partial s} \phi & =\epsilon^{-c} \frac{\partial w}{\partial \tau} \frac{\partial \tau}{\partial s} \\
& =\epsilon^{b-c} \frac{\partial w}{\partial \tau}
\end{aligned}
$$

and

$$
\begin{aligned}
-2 \sqrt{x} \frac{\partial}{\partial x} \sqrt{x} \phi & =-2 \sqrt{x}\left(\frac{\partial \sqrt{x}}{\partial x} \phi+\sqrt{x} \frac{\partial \phi}{\partial x}\right) \\
& =-2 \sqrt{x} \frac{1}{2 \sqrt{x}} \phi-2 \sqrt{x} \sqrt{x} \frac{\partial \phi}{\partial x} \\
& =-\phi-2 x \frac{\partial \phi}{\partial x}
\end{aligned}
$$

where

$$
\begin{aligned}
\frac{\partial \phi}{\partial x} & =\epsilon^{-c} \frac{\partial w}{\partial \xi} \frac{\partial \xi}{\partial x} \\
& =\epsilon^{a-c} \frac{\partial w}{\partial \xi} .
\end{aligned}
$$

By using Eqs. (142)-(144) in Eq. (32), the RZSE is then written

$$
\epsilon^{-c}\left[\epsilon^{b} \frac{\partial w}{\partial \tau}+w+2 \xi \frac{\partial w}{\partial \xi}\right]=0
$$

and is invariant under the transformation $\forall \epsilon$ if $\epsilon^{b}=2$, i.e.,

$$
\epsilon^{-c}\left[\frac{\epsilon^{b}}{2}\left(\frac{\partial w}{\partial \tau_{\Re}}-i \frac{\partial w}{\partial \tau_{\Im}}\right)+w+2 \xi \frac{\partial w}{\partial \xi}\right]=0,
$$

and

$$
b=\frac{\log (2)+2 i \pi n}{\log (\epsilon)}, \forall n \in \mathbb{Z} .
$$

Therefore, it can be seen that since $\phi$ solves the RZSE for $x$ and $s$, then $w=\epsilon^{-c} \phi$ solves the RZSE at $x=\epsilon^{-a} \xi$, and $s=\epsilon^{-b} \tau$. We now construct a group of independent variables such that

$$
\begin{aligned}
\frac{\xi}{\tau^{a / b}} & =\frac{\epsilon^{a} x}{\left(\epsilon^{b} s\right)^{a / b}} \\
& =\frac{x}{s^{a / b}} \\
& =\eta(x, s),
\end{aligned}
$$

and the similarity variable is then

$$
\eta(x, s)=x s^{-\frac{a \log (\epsilon)}{\log (2)+2 i \pi n}}
$$

Also,

$$
\begin{aligned}
\frac{w}{\tau^{c / b}} & =\frac{\epsilon^{c} \phi}{\left(\epsilon^{b} s\right)^{c / b}} \\
& =\frac{\phi}{s^{c / b}} \\
& =\nu(\eta),
\end{aligned}
$$

suggesting that we seek a solution of the RZSE with the form

$$
\phi_{s}(x)=s^{\frac{c \log (\epsilon)}{\log (2)+2 i \pi n}} \nu(\eta) .
$$


Since the RZSE is invariant under the transformation, it is to be expected that the solution will also be invariant under the variable transformation. Taking $a=c=\log ^{-1}(\epsilon)$, the partial derivatives transform like

$$
\begin{aligned}
\frac{\partial}{\partial s} \phi_{s}(x) & =\frac{\partial}{\partial s}\left(s^{\frac{1}{\log (2)+2 i \pi n}}\right) \nu(\eta)+\left(s^{\frac{1}{\log (2)+2 i \pi n}}\right) \nu^{\prime}(\eta) \frac{\partial \eta}{\partial s} \\
& =\frac{s^{-1+\frac{1}{\log (2)+2 i \pi n}}}{\log (2)+2 i \pi n}\left[\nu(\eta)-\nu^{\prime}(\eta)\right],
\end{aligned}
$$

and

$$
\begin{aligned}
\frac{\partial}{\partial x} \phi_{s}(x) & =\left(s^{\frac{1}{\log (2)+2 i \pi n}}\right) \nu^{\prime}(\eta) \frac{\partial \eta}{\partial x} \\
& =\nu^{\prime}(\eta)
\end{aligned}
$$

where

$$
\frac{\partial \eta}{\partial s}=-\frac{s^{-1}}{2 i \pi n+\log (2)}
$$

and

$$
\frac{\partial \eta}{\partial x}=s^{-\frac{1}{2 i \pi n+\log (2)}}
$$

The RZSE then reduces to the ODE

$$
\left[s^{-1}+\log (2)+2 i \pi n\right] \nu(\eta)+\left[-s^{-1}+2 \log (2) \eta+4 i \pi n \eta\right] \nu^{\prime}(\eta)=0, \forall n \in \mathbb{Z} .
$$

\section{A. General Solution}

The homogenous linear differential Eq. (156) is separable [35, viz.,

$$
\frac{d \nu}{\nu}=\frac{2 i \pi n+s^{-1}+\log (2)}{s^{-1}-4 i \pi n \eta-\eta \log (4)} d \eta
$$

Integrating on both sides, we obtain

$$
\ln |\nu|=c_{1}-\frac{\left(2 i \pi n+s^{-1}+\log (2)\right) \log \left(s^{-1}-4 i \pi n \eta-\eta \log (4)\right)}{4 i \pi n+\log (4)} .
$$

Exponentiating both sides,

$$
|\nu|=\exp \left(c_{1}\right)\left(s^{-1}-4 i \pi n \eta-\eta \log (4)\right)^{-\frac{2 i \pi n+s^{-1}+\log (2)}{4 i \pi n+\log (4)}} .
$$

Renaming the constant $\exp \left(c_{1}\right)=C$ and dropping the absolute value recovers the lost solution $\nu(\eta)=0$, giving the general solution to Eq. 156

$$
\nu_{n}(\eta)=C\left(s^{-1}-4 i \pi n \eta-\eta \log (4)\right)^{-\frac{2 i \pi n+s^{-1}+\log (2)}{4 i \pi n+\log (4)}}, \forall n \in \mathbb{Z}, \forall C \in \mathbb{R} .
$$

By setting $C=1$, and using Eqs. (149) and 151) in Eq. (160), we obtain the general solution to the RZSE Eq. 32, written

$$
\phi_{s}(x)=s^{\frac{1}{\log (2)+2 i \pi n}}\left[\frac{1}{s}+s^{-\frac{1}{\log (2)+2 i \pi n}}(-x \log (4)-4 i \pi n x)\right]^{-\frac{2 \pi n s+i s \log (2)+i}{4 \pi n s-i s \log (4)}}, \forall n \in \mathbb{Z} .
$$




\section{CONCLUSION}

In this study, we have discussed the convergence of the real part of every nontrivial zero of the analytic continuation of the Riemann zeta function. This was accomplished by developing a Riemann zeta Schrödinger equation and comparing it with the Bender-Brody-Müller conjecture in both configuration space and momentum space. A symmetrization procedure was implemented to study the convergence of the system, and the expectation values were calculated from the resulting system to study the nontrivial zeros of the analytic continuation of the Riemann zeta function. Moreover, a second quantization procedure was performed for the Riemann zeta Schrödinger equation to obtain the equations of motion and an analytical expression for the eigenvalues. It was also demonstrated that the eigenvalues are holomorphic. A normalized convergent expression for the analytic continuation of the nontrivial zeros of the Riemann zeta function was obtained, and a convergence test for the expression was performed demonstrating that the real part of every nontrivial zero of the Riemann zeta function converges at $\sigma=1 / 2$. Finally, a general solution to the Riemann zeta Schrödinger equation was found from performing an invariant similarity transformation.

[1] H.C. Rosu, Modern Physics Letters A 18(18), 1205-1213 (2003).

[2] D. Schumayer, B.P. van Zyl, and D.A. Hutchinson, Physical Review E 78(5), 056215 (2008).

[3] B.L. Julia, Physica A: Statistical Mechanics and its Applications 203(3 - 4), 425-436 (1994).

[4] D. Spector, Journal of Mathematical Physics 39(4), 1919-1927 (1998).

[5] D. Schumayer and D.A. Hutchinson, Reviews of Modern Physics 83(2), 307 (2011).

[6] P. Ribenboim, The little Book of Big Primes, (1991).

[7] C.M. Bender, D.C. Brody and M.P. Müller, Physical Review Letters 118(13) 130201 (2017).

[8] B. Riemann, On the Number of Prime Numbers less than a Given Quantity.

[9] W.G. Faris and R.B. Lavine, Communications in Mathematical Physics 35(1), 39-48 (1974).

[10] D. Hilbert, Bulletin of the American Mathematical Society 8(10), 437-479 (1902).

[11] C.M. Bender, S. Boettcher and P.N. Meisinger, Journal of Mathematical Physics 40(5) 2201-2229 (1999).

[12] M.V. Berry and J.P. Keating, In Supersymmetry and Trace Formulae, Springer US,355-367 (1999).

[13] M.V. Berry and J.P. Keating, SIAM review 41(2), 236-266 (1999).

[14] A. Connes, Selecta Mathematica, New Series 5(1) 29-106 (1999).

[15] G. Sierra and J. Rodriguez-Laguna, Physical review letters 106(20) 200201 (2011).

[16] M.V. Berry and J.P. Keating, Journal of Physics A: Mathematical and Theoretical 44(28) 285203 (2011).

[17] G. Sierra, Journal of Physics A: Mathematical and Theoretical 47(32) 325204 (2014).

[18] A.M. Odlyzko, Contemporary Mathematics 290139 (2001).

[19] J.C. Andrade, International Journal of Modern Physics A 2850072 (2013).

[20] E.C. Titchmarsh and D.R. Heath-Brown, Oxford University Press (1986).

[21] G.H. Hardy, CR Acad. Sci. Paris 158, 1012-1014 (1914).

[22] J. Hadamard, Bull. Soc. Math. France 24199 (1896).

[23] C. J. de la Vallée-Poussin, Ann. Soc. Sci. Bruxelles 20, 183 (1896).

[24] Whittaker, E.T. and Watson, G.N., 1996. A Course of Modern Analysis. Cambridge University Press.

[25] O. Espinosa and V.H. Moll, The Ramanujan Journal 6(2), 159 (2002).

[26] G. Sierra, Nuclear Physics B 776(3) 327-364 (2007).

[27] J. Twamley and G.J.Milburn, New Journal of Physics 8(12), 328 (2006).

[28] S. Endres and F. Steiner, Journal of Physics A: Mathematical and Theoretical 43(9) 095204 (2010).

[29] T. Kato, Transactions of the American Mathematical Society 70(2) 195 (1951).

[30] M. Plancherel and M. Leffler, Rendiconti del Circolo Matematico di Palermo 30(1) 289 (1884).

[31] Reed, M. and Simon, B., Methods of Modern Mathematical Physics: Functional analysis (1) (1980).

[32] J.M. Cook, Transactions of the American Mathematical Society 74(2) 222 (1953).

[33] M. Abramowitz and I.A. Stegun, Handbook of Mathematical Functions with Formulas, Graphs, and Mathematical Tables, 9th printing. New York: Dover 358 (1972).

[34] M. Pakdemirli and M. Yurusoy, SIAM review, 40(1) 96 (1998).

[35] J.L. Massera and J.J. Schaffer, Annals of mathematics 517 (1958). 


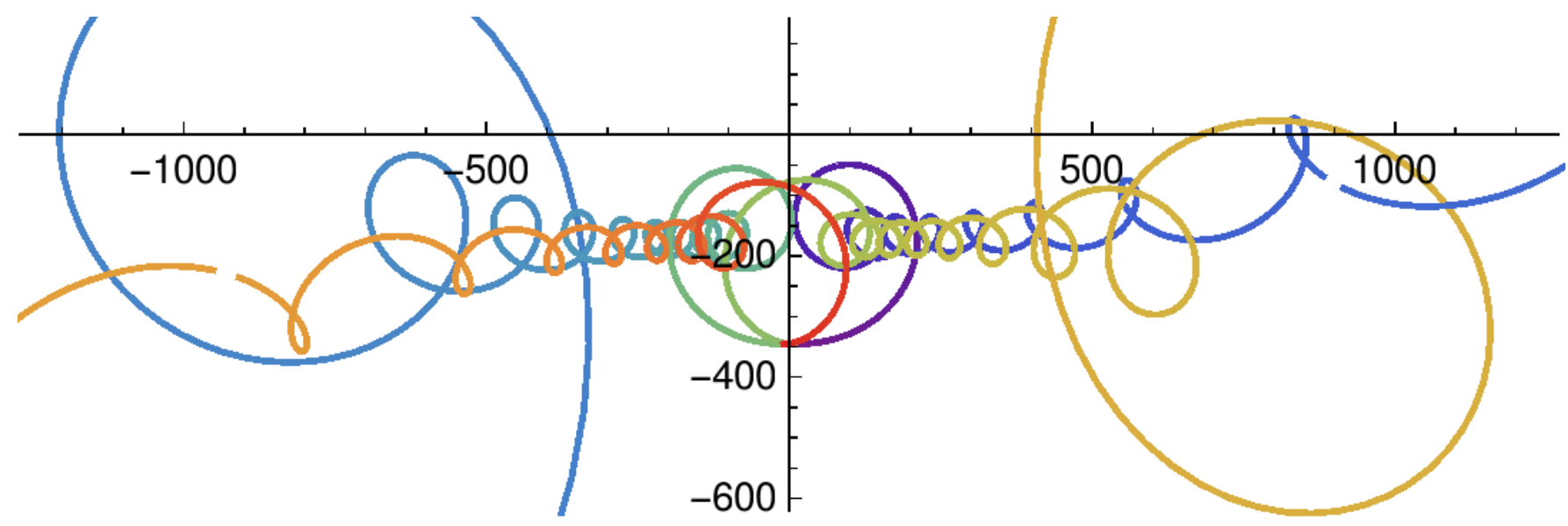

Figure 2: Zoom in of the parametric plot of the eigenvalues in Eq. 139 with coordinates $\Re\left(t_{n}\right)$ and $\Im\left(t_{n}\right)$ as a function of $\theta$. The lower bound of the summation is $n=0$ and the upper bound of the summation is $n=10$ (color online). 